\title{
Experimental Verification of Optimized Multiscroll Chaotic Oscillators Based on Irregular Saturated Functions
}

\author{
J. M. Muñoz-Pacheco $\mathbb{D}^{\mathbb{D}},{ }^{1}$ D. K. Guevara-Flores, ${ }^{1}$ O. G. Félix-Beltrán, ${ }^{1}$ E. Tlelo-Cuautle $\mathbb{D}^{\mathbb{D}},{ }^{2}$ \\ J. E. Barradas-Guevara, ${ }^{3}$ and C. K. Volos ${ }^{4}$ \\ ${ }^{1}$ Faculty of Electronics Sciences, Autonomous University of Puebla, 72570 Puebla, PUE, Mexico \\ ${ }^{2}$ Department of Electronics, National Institute of Astrophysics, Optics and Electronics, 72840 Tonantzintla, PUE, Mexico \\ ${ }^{3}$ Faculty of Physics and Mathematics Sciences, Autonomous University of Puebla, 72570 Puebla, PUE, Mexico \\ ${ }^{4}$ Laboratory of Nonlinear Systems, Circuits \& Complexity, Department of Physics, Aristotle University of Thessaloniki, \\ 54124 Thessaloniki, Greece \\ Correspondence should be addressed to J. M. Muñoz-Pacheco; jesusm.pacheco@correo.buap.mx
}

Received 1 October 2017; Revised 25 December 2017; Accepted 30 January 2018; Published 6 March 2018

Academic Editor: Kevin Wong

Copyright (C) 2018 J. M. Muñoz-Pacheco et al. This is an open access article distributed under the Creative Commons Attribution License, which permits unrestricted use, distribution, and reproduction in any medium, provided the original work is properly cited.

\begin{abstract}
Multiscroll chaotic attractors generated by irregular saturated nonlinear functions with optimized positive Lyapunov exponent are designed and implemented. The saturated nonlinear functions are designed in an irregular way by modifying their parameters such as slopes, delays between slopes, and breakpoints. Then, the positive Lyapunov exponent is optimized using the differential evolution algorithm to obtain chaotic attractors with 2 to 5 scrolls. We observed that the resulting chaotic attractors present more complex dynamics when different patterns of irregular saturated nonlinear functions are considered. After that, the optimized chaotic oscillators are physically implemented with an analog discrete circuit to validate the use of proposed irregular saturated functions. Experimental results are consistent with MATLAB $^{\mathrm{TM}}$ and SPICE circuit simulator. Finally, the synchronization between optimized and nonoptimized chaotic oscillators is demonstrated.
\end{abstract}

\section{Introduction}

The chaotic behavior has attracted a lot of attention for scientific community due to extreme sensitivity to its initial conditions and the broadband nature of its chaotic signals [1-28]. Therefore, in the last years, literature is vast in papers oriented to study new chaotic systems [1-5], propose novel applications [6-9], increase the degree of chaos (hyperchaotic systems $[8,10,11])$, get fractional order chaotic systems $[7$, $12,13]$, synchronize the chaotic behavior [14-18], optimize chaotic systems [19-22], and implement chaotic oscillators using electronic circuits [23-28]. In all these studies, chaos behavior is analyzed and verified by using different approaches, for example, frequency spectrum, Poincaré maps, bifurcation diagrams, Lyapunov exponents, and stability of equilibrium points. Among them, Lyapunov exponents provide a direct measure of the sensitive dependence on initial conditions by quantifying the exponential rates at which neighboring orbits on an attractor diverge as the system evolves in time [29-31].

For an $n$-dimensional nonlinear system, if the system has at least one positive Lyapunov exponent (LE) and is purely deterministic, then it is chaotic. Indeed, a tool commonly used to determine the presence of chaos in several numerical and experimental results is to compute only the positive LE [31].

Besides, the positive LE can be very useful to determine the unpredictability grade of the chaotic oscillator because its magnitude specifies the maximum average exponential rate corresponding to divergence of trajectories on an attractor and thus the maximum amount of instability along any direction [29-31]. That is, a high value of the positive LE can be taken as an indication of a high degree of chaos in the dynamical system [19-22, 32-35]. 
For instance, in [32] the speed effects and leg amputations on the dynamic stability of running are analyzed by computing the largest LE. The results revealed that the value of the largest LE is positive not only for unaffected patients but also for the one-leg affected patients. However, the positive LE of embedded time-series data from the affected leg was higher than for the unaffected leg indicating a more rich dynamics. In [33] a numerical scheme based on the Lattice Boltzmann method for the flow in complex mixer geometries to compute trajectories of passive tracers for the quantification of chaotic mixing was reported. They reported a better efficiency in chaotic micromixers when the value of positive LE was higher. Moreover, chaotic systems with a high value of the positive LE have also been used to improve the performance of chaos-based applications, for example, in [34] it was demonstrated that a high value of the positive LE in an optimization algorithm based on a particle swarm implies that the particles are inclined to explore different regions and find better fitness values. Therefore, the particle swarm with just a little variation in the value of positive LE usually achieved a better performance, especially for multimodal functions. In [35] the efficiency of hybrid chaotic optimization algorithms was studied by revealing effects on the search speed as a function of chaotic sequences from different chaotic maps. It was found that the higher the magnitude of positive LE, the faster the search speed in whole optimization space. Accordingly, the efficiency of global optimization was directly proportional to the value of positive LE.

In this framework, optimized chaotic systems with a high value of the positive LE can be extremely suitable to enhance the existing chaos-based applications. In electronics, a great variety of multiscroll chaotic oscillators has been implemented with commercially available electronic devices, as well as with integrated circuits technology $[5,10,23,26-$ $28,36,37]$. However, those experimental realizations are not optimized to provide a high value of the positive Lyapunov exponent (PLE). Although some authors have already used optimization algorithms based on evolutionary computation to get optimized multiscrolls chaotic systems [19, 22], they were obtained by using piecewise-linear (PWL) functions in the form of saturated nonlinear functions (SNLF) with symmetry properties. In addition, the experimental verification of those approaches is lacking.

This paper is motivated by the aforementioned discussion. In that scenario, we design irregular SNLF to obtain multiscroll chaotic attractors with optimized values of the positive LE. Additionally, we also demonstrate its practical feasibility by the physical implementation of the resulting multiscroll chaotic oscillators. Two cases were considered to design the irregular SNLFs. The first one consists of changing the breakpoints of SNLF to get different slopes, whereas the second one modifies the delay between slopes in different sections of SNLF. In both cases, once the parameters of SNLF are defined, we apply the evolutionary algorithms reported in $[19,22]$ to find the optimal value for system's parameters which maximizes the magnitude of positive LE. As a result, multiscroll chaotic oscillators with a more complex dynamics are generated. Experimental results for 2-, 3-, 4-, and 5scroll chaotic attractors were obtained with the aim of an analog discrete circuit based on commercial operational amplifiers (OpAmps). Further, we show the synchronization of those optimized chaotic oscillators by using generalized Hamiltonian forms because they can enhance the synchronization and realization of secure communication systems, for instance.

The paper is organized as follows. Section 2 describes the multiscroll chaotic oscillator under study; Section 3 outlines the steps to obtain optimized values of positive LE as well as the electronic design. Sections 4 and 5 present the experimental confirmation of the proposed approach for the two cases: slopes varying and different delays between slopes, respectively. Section 6 demonstrates the synchronization between optimized and nonoptimized chaotic oscillators. Finally, conclusions are given in Section 7.

\section{SNLF-Based Multiscroll Chaotic Oscillator}

The case of study in this work is the chaotic oscillator described by

$$
\begin{aligned}
& \dot{x}=y, \\
& \dot{y}=z, \\
& \dot{z}=-a x-b y-c z+d_{1} f(x ; q),
\end{aligned}
$$

where $(x, y, z)$ is the state variables, $f(x ; q)$ is the SNLF, and $\left(a, b, c, d_{1}\right)$ is the system's parameters. To maximize the value of positive LE requires varying the coefficients of chaotic oscillator, leading to a huge number of combinations. Herein, system's parameters $\left(a, b, c, d_{1}\right)$ are varied within the range $[0.0001 \cdots 1.0000]$. So, we define four variables where each one can have $\left(2 \times 10^{4}\right)^{4}=16 \times 10^{16}$ possible combinations. This result justifies the application of heuristics like the ones already introduced in $[19,22]$. For all cases analyzed in this work, the phase-space plots show the state variables $x$ versus $y$.

The first step consists of manipulating $f(x ; q)$ with the goal of incrementing the complexity of multiscroll chaotic oscillator. To generate 2 scrolls, the SNFL description is given by

$$
f(x ; q)= \begin{cases}k, & \text { if } x>q(2), \\ \frac{k}{q}, & \text { if } q(2)>x>q(1), \\ -k, & \text { if } x<q(1),\end{cases}
$$

where $k$ represents the value of saturated regions, $k v=k / q$ is the slope between two saturated regions, $q$ is a break point connecting a saturated region with a slope, and $h$ is the delay, as shown in Figure 1. In general, the number of scrolls to be generated equals the number of saturated regions $k$. Therefore, (2) can be augmented to generate $n$-scrolls as shown in [38]. It means that by augmenting segments in a symmetric way as shown in Figures 1(a) and 1(b), respectively, even and odd number of scrolls are generated. However, in 


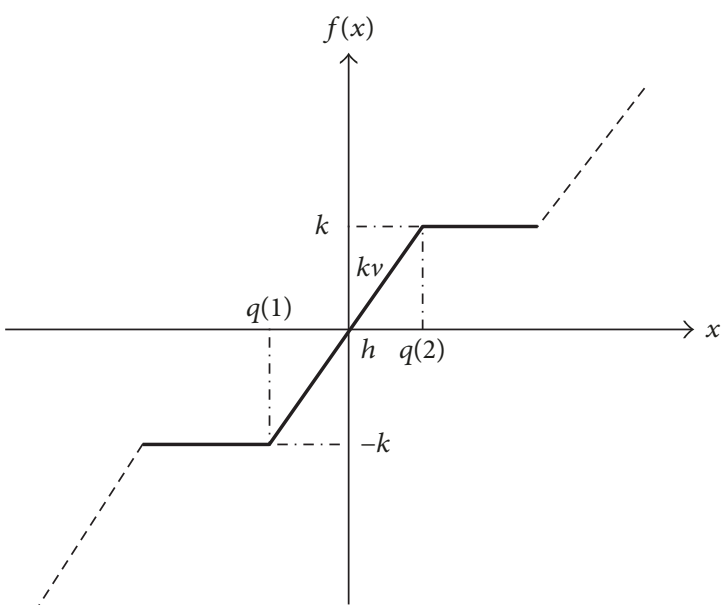

(a)

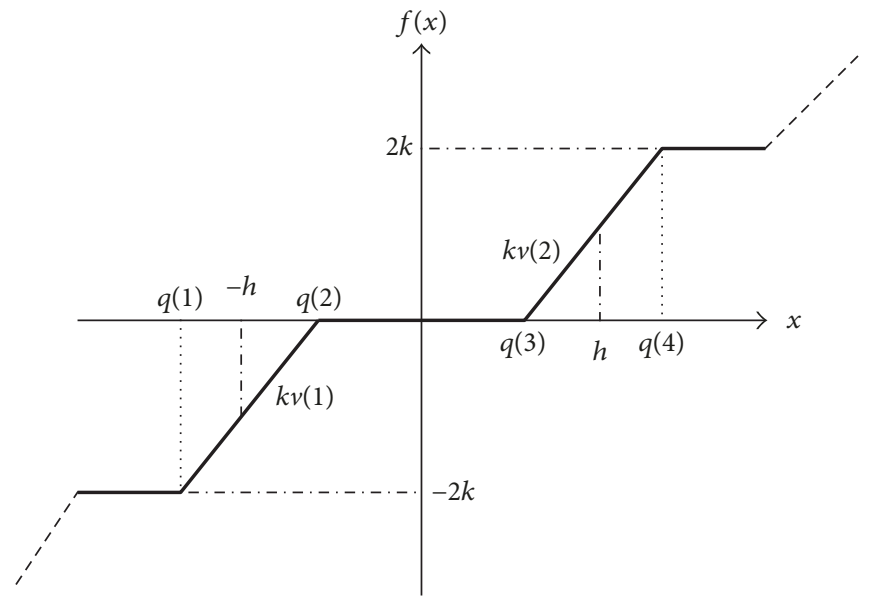

(b)

FIGURE 1: Description of a typical SNLF function for generating (a) even and (b) odd scrolls.

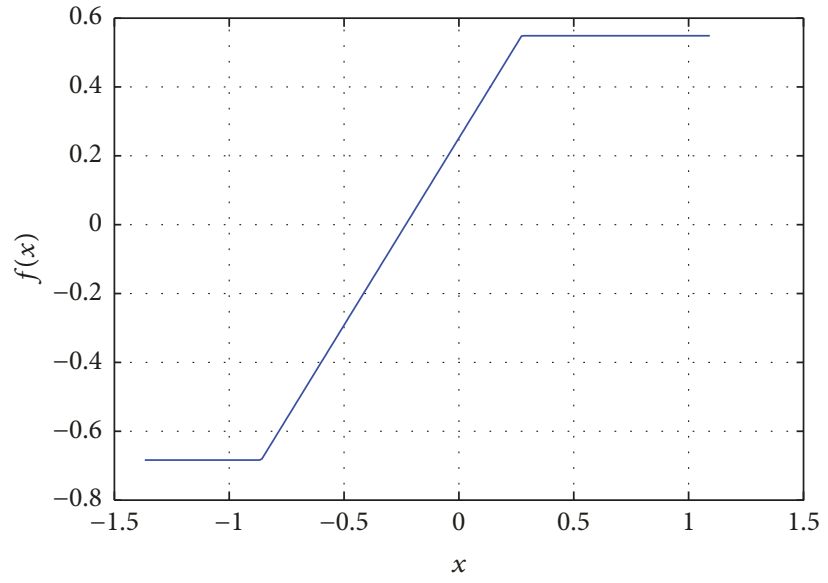

(a)

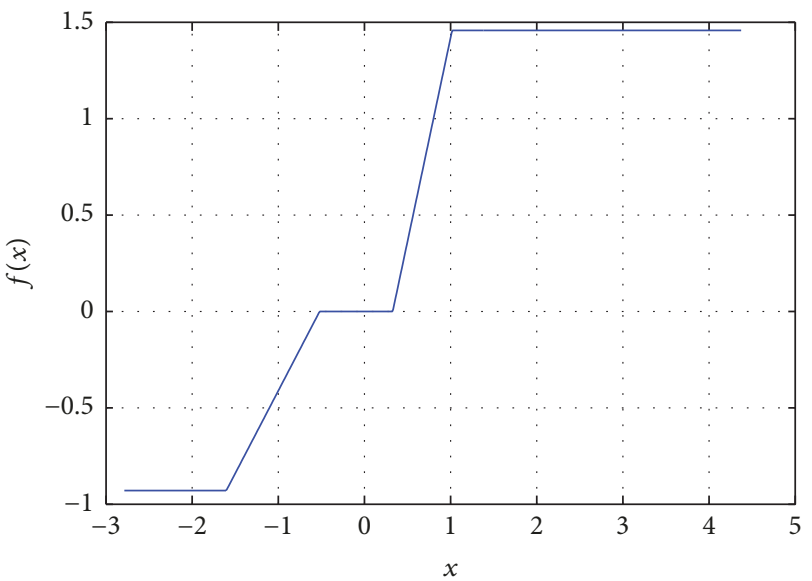

(b)

FIGURE 2: Examples of irregular SNLFs with (a) 2 and (b) 3 saturated levels.

this work we show how to use irregular SNLF functions, that is, nonsymmetric, to obtain multiscrolls as the ones shown in Figure 2. The irregular SNLF functions are herein designed using different values for breakpoints $q(l)$ with $l=$ $1,2,3, \ldots, j$ where $j$ is the number of breakpoints, slopes $k v$, and saturated levels $k$.

\section{Methodology to Maximize the Positive LE of Multiscrolls Oscillators and Its Circuit Design}

The solution to (1) by using symmetric SNLF described by (2) has been performed by applying evolutionary algorithms in $[19,22]$, where the positive LE was optimized. By applying the differential evolution (DE) algorithm already introduced in [22], we obtained several feasible solutions for the coefficients $a, b, c$, and $d_{1}$, which provide higher values of the positive
LE as the number of scrolls is incremented. In all cases, the value of positive $\mathrm{LE}$ computed by $\mathrm{DE}$ is higher than when using traditional values (TV) for system's parameters as TV $=$ $\left\{a=b=c=d_{1}=0.7\right\}$ [19]. Details of applying DE algorithm to optimize the positive LE can be found in [22].

On the other hand, the SNLF can be implemented as shown in Figure 3, where the number of saturated levels (SL) determines the number of OpAmps to be used considering $\#$ OpAmp $=\#$ SL-1, as shown in [23]. In that reference the whole electronic realization of (1) is given by using OpAmps. That realization is redrawn in Figure 4, where the SNLF function is embedded in block PWL, and system's parameters $a, b, c$, and $d_{1}$ are implemented by the ratio of resistors $R_{f a} / R_{i a}, R_{f b} / R_{i b}, R_{f c} / R_{\mathrm{ic}}$, and $R_{f d} / R_{i d}$, respectively.

Otherwise, this article shows how realizing irregular SNLFs by modifying Figure 3, such as asymmetric variation of the slopes, modifying the breakpoints $q(l), l=$ $1, \ldots, m$, and asymmetric variation of the saturated levels $k$. 


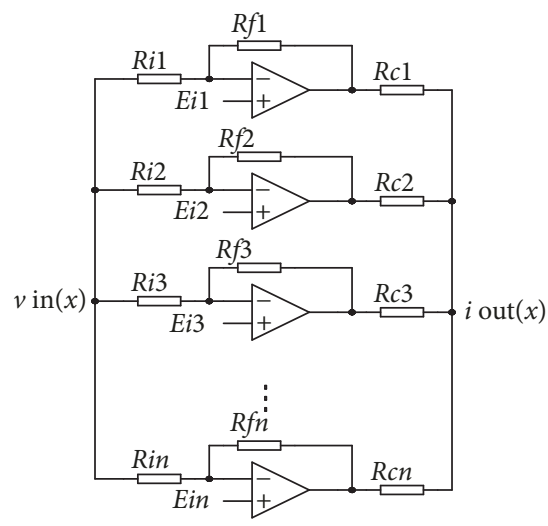

FIgURE 3: Realization of SNLFs using OpAmps represented in Figure 4 as PWL block.

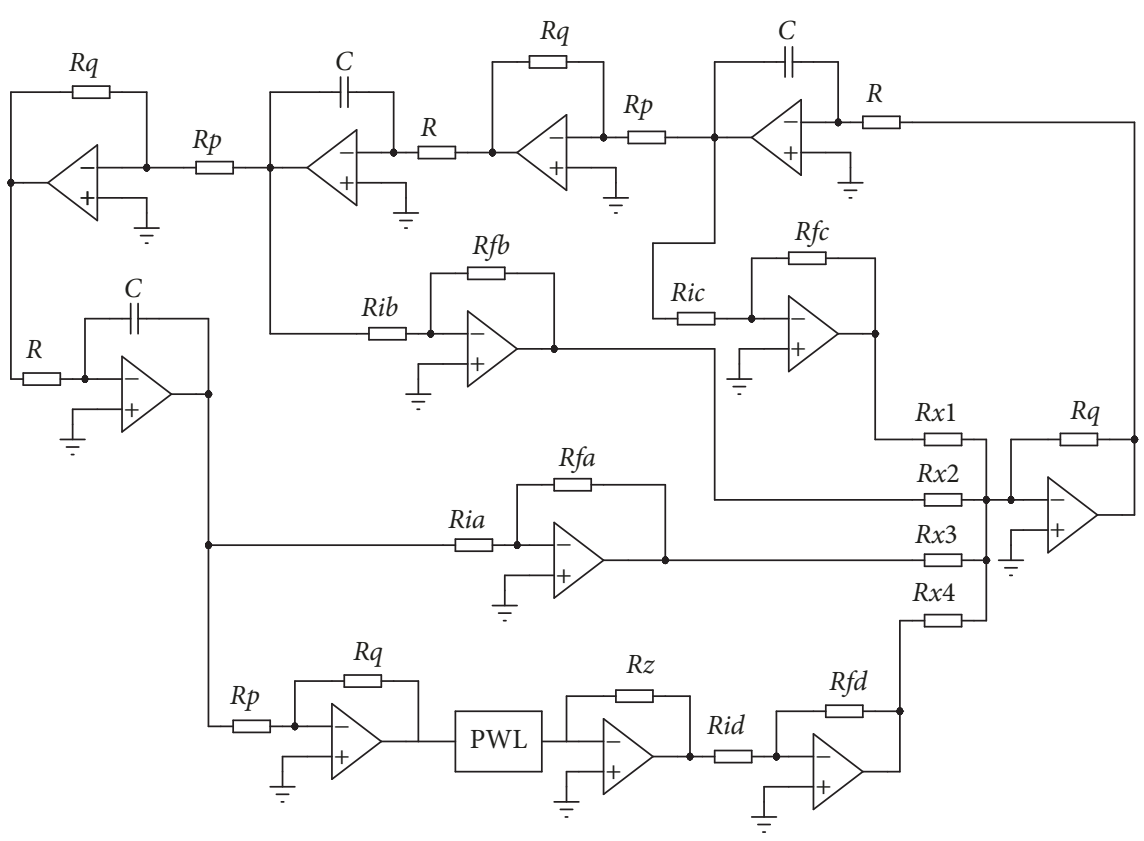

FIgURE 4: Realization of (1) using OpAmps.

The experiments are performed considering the following steps:

(1) Modify the SNLFs to obtain an irregular one using MATLAB.

(2) Optimize the value of positive LE by computing the optimal values for $a, b, c$, and $d_{1}$ with the DE algorithm given in [22].

(3) Implement physically the SNLFs with OpAmps.

(4) Validate experimentally the optimized chaotic oscillator by generating the required number of scrolls.

For instance, the MATLAB, SPICE, and experimental results of a 5-scrolls chaotic attractor obtained with a irregular SNLF are shown in Figure 5. More details are given in the following sections.

\section{Experimental Results by Varying the Slopes of SNLFs}

The slope in SNLF is described herein by $k v$, as shown in Figure 1, which is modified asymmetrically to generate irregular SNLFs for obtaining 3, 4, and 5 scrolls. The first step consists of determining the minimum and maximum slopes values using MATLAB, so that the results to attain from 2 to 7 scrolls are given in Table 1. To generate more scrolls, the minimum and maximum values are the same as for 6 and 7 scrolls. Next, the values of circuit elements to get different slopes in SNLF are found. This is done by using commercially available OpAmps in Figure 3. Table 2 lists the calculated values to obtain slopes of $5,10,20,30$, 40,50 , and 100 . In all cases it is assumed a saturation voltage $V_{\text {sat }}=15 \mathrm{~V}$ for the OpAmps. Using Table 2, one can combine 
TABLE 1: Values of the slopes computed by MATLAB.

\begin{tabular}{lcc}
\hline Scrolls & Minimum slope $k v$ & Maximum slope $k v$ \\
\hline 2 & 2 & $\infty$ \\
3 & 4 & $\infty$ \\
4 & 5 & $\infty$ \\
5 & 7 & $\infty$ \\
6 & 10 & $\infty$ \\
7 & 10 & $\infty$ \\
\hline
\end{tabular}

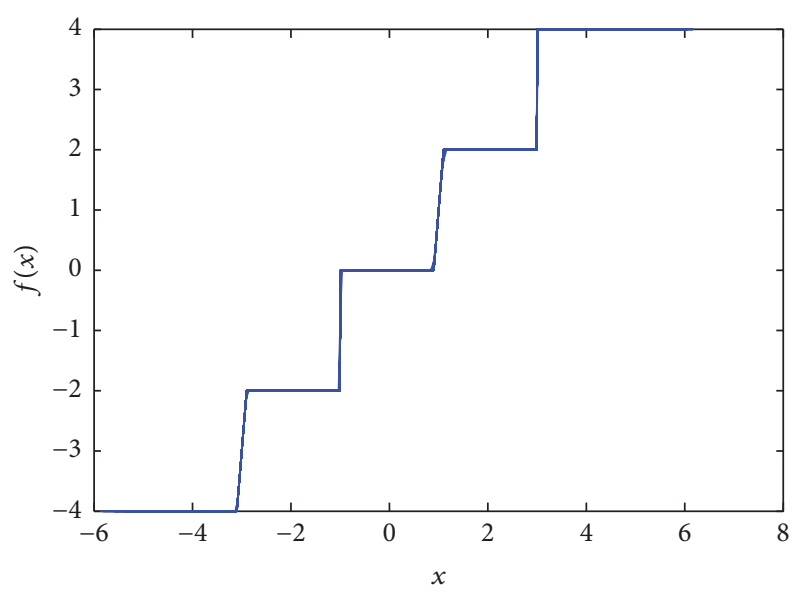

(a)

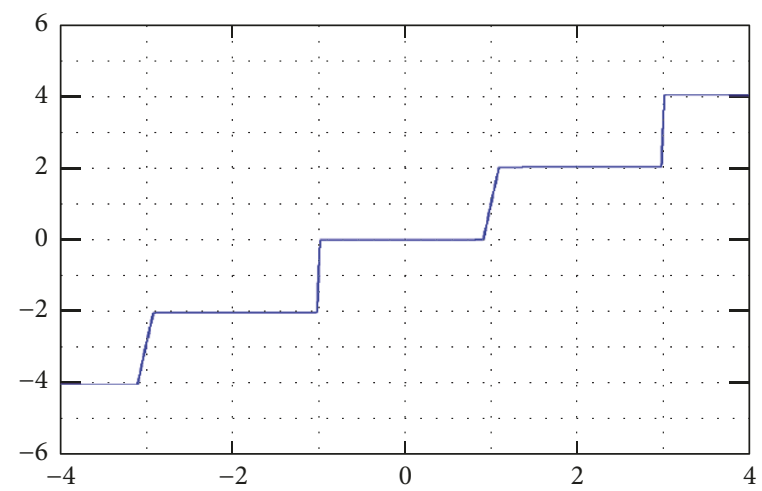

(c)

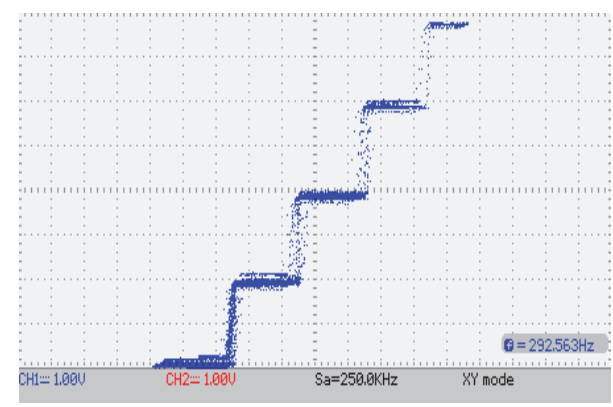

(e)

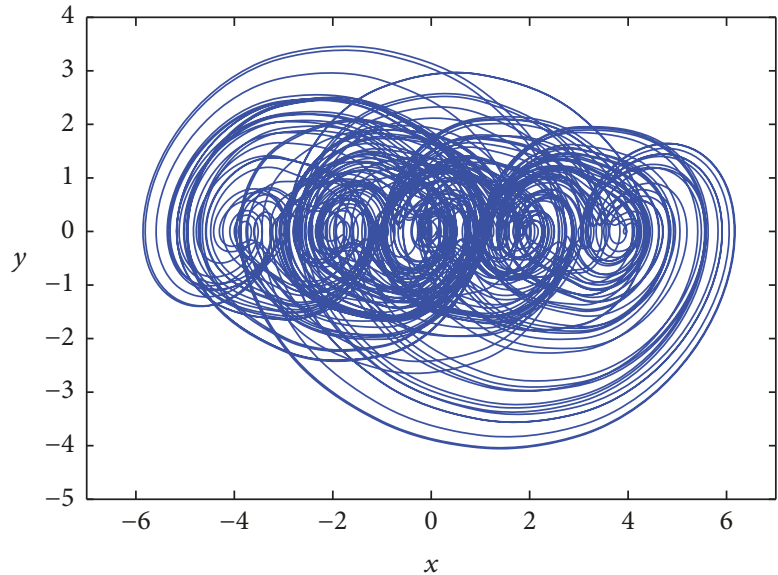

(b)

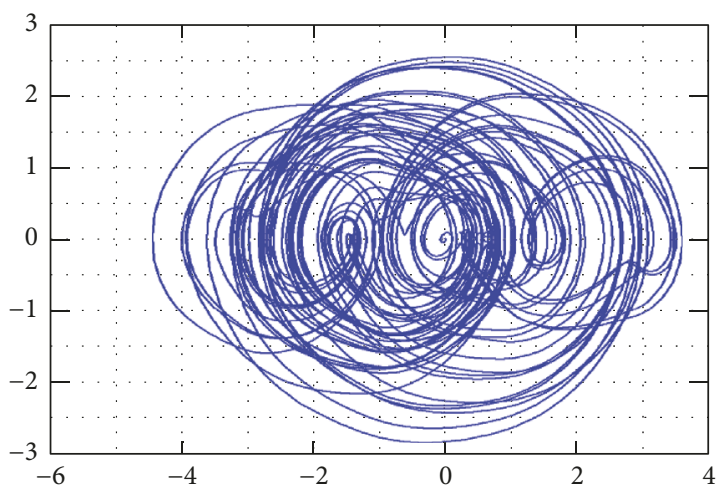

(d)

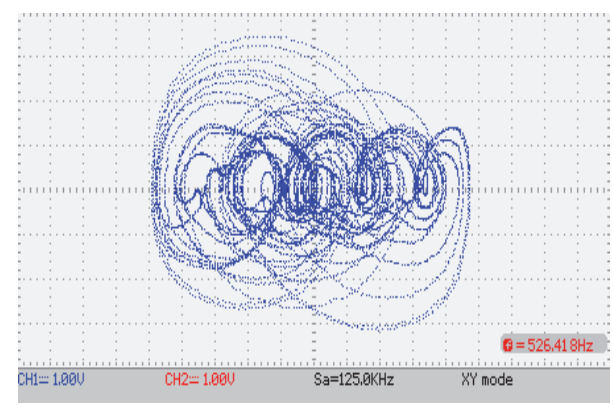

(f)

FIGURE 5: 5-scroll using irregular SNLF for parameters given in Table 2: irregular SNLF and chaotic attractor with (a), (b) MATLAB, (c), (d) SPICE, and (e), (f) experimental realization. 
TABLE 2: Circuit element values for implementing different slopes using Figure 3.

\begin{tabular}{lccc}
\hline Slope value & $R_{i}(\mathrm{k} \Omega)$ & $R_{f}(\mathrm{k} \Omega)$ & $R_{c}(\mathrm{k} \Omega)$ \\
\hline 5 & 1.0 & 75 & 150 \\
10 & 1.0 & 150 & 150 \\
20 & 1.0 & 300 & 150 \\
30 & 1.0 & 450 & 150 \\
40 & 1.0 & 600 & 150 \\
50 & 1.0 & 750 & 150 \\
100 & 1.0 & 1500 & 150 \\
\hline
\end{tabular}

TABLE 3: Combinations of different slopes for realizing irregular SNLFs generating 3 to 5 scrolls.

\begin{tabular}{lccc}
\hline Scrolls & & Case & 3 \\
\hline 3 & 1 & 2 & {$[20,50]$} \\
4 & {$[5,10]$} & {$[10,20]$} & {$[30,50,70]$} \\
5 & {$[10,10,5]$} & {$[50,10,20]$} & {$[10,100,10,100]$} \\
\hline
\end{tabular}

TABLE 4: Positive LEs for the optimized multiscrolls chaotic oscillators with different slopes $k v$ as the cases listed in Table 3.

\begin{tabular}{|c|c|c|c|c|c|c|c|}
\hline Case & Scroll number & $\begin{array}{l}\text { Positive LE without } \\
\text { optimization }\end{array}$ & $a$ & $b$ & $c$ & $d_{1}$ & $\begin{array}{c}\text { Positive LE with optimization } \\
\text { and irregular SNLF }\end{array}$ \\
\hline \multirow{3}{*}{1} & 3 & 0.2578 & 1.000 & 0.751 & 0.394 & 0.981 & 0.5555 \\
\hline & 4 & 0.2890 & 1.000 & 0.709 & 0.290 & 1.000 & 0.6327 \\
\hline & 5 & 0.3279 & 1.000 & 0.781 & 0.170 & 1.000 & 0.6580 \\
\hline \multirow{3}{*}{2} & 3 & 0.2737 & 0.997 & 0.783 & 0.395 & 0.988 & 0.5608 \\
\hline & 4 & 0.3121 & 1.000 & 0.745 & 0.304 & 1.000 & 0.6470 \\
\hline & 5 & 0.2763 & 1.000 & 0.658 & 0.262 & 0.999 & 0.6823 \\
\hline \multirow{3}{*}{3} & 3 & 0.2651 & 1.000 & 1.000 & 0.270 & 0.998 & 0.5656 \\
\hline & 4 & 0.2760 & 1.000 & 0.927 & 0.189 & 1.000 & 0.6667 \\
\hline & 5 & 0.3016 & 1.000 & 0.680 & 0.262 & 1.000 & 0.7112 \\
\hline
\end{tabular}

different slopes to realize irregular SNLFs. In this manner, Table 3 shows the combinations of slopes to generate 3 , 4 , and 5 scrolls. In the experiments, those values have been selected randomly from Table 2. Each combination of slopes is used to implement the irregular SNLF described by (2) and then to implement the dynamical system described in (1). All cases in Table 3 for generating 3-, 4-, and 5-scroll chaotic attractors with optimized positive LE are described below.

4.1. Optimized 3-Scrolls Chaotic Oscillator with Different Slopes. Using the irregular SNLF with the slopes listed in Table 3 and setting traditional values of $a=b=c=d_{1}=0.7$, the positive LE is listed in Table 4. This is positive for the three cases indicating chaotic behavior. Afterwards, by applying DE algorithm [22] the optimized positive LE provides new coefficient values that are also listed in Table 4. As supposed the value of positive LE is higher than those values without optimization. The optimization of positive LE applying [22] was executed using a population of 50 individuals and 50 iterations.

According to case 1 from Table 3 and from Figure 1, the generation of 3 scrolls with optimized positive LE implies slopes being $[5,10]$, which are located in the third and first quadrants, respectively. Cases 2 and 3 require the slopes being $[10,20]$ and $[20,50]$, respectively. The circuit element values for realizing these slopes were already listed in Table 2. Figure 6 shows the experimental results for the irregular SNLF for each case and the associated attractor showing 3 scrolls with optimized positive LE.

4.2. Optimized 4-Scroll Chaotic Oscillator with Different Slopes. For generating 4 scrolls with optimized positive LE, the values of the slopes are also listed in Table 3. Again, by setting traditional values $a=b=c=d_{1}=0.7$, positive $\mathrm{LE}$ is listed in Table 4. As a result, it is more positive for the three cases than those values computed for 3 scrolls in Table 4 . This confirms that positive LE increases by augmenting the number of scrolls.

The optimized positive LE provides new values for system's parameters that are listed in Table 4. As can be appreciated, the optimized positive LE is also higher for each case than for 3 scrolls. The optimization for positive LE applying [22] was executed using a population of 50 individuals and 50 iterations. The irregular SNLFs are realized 

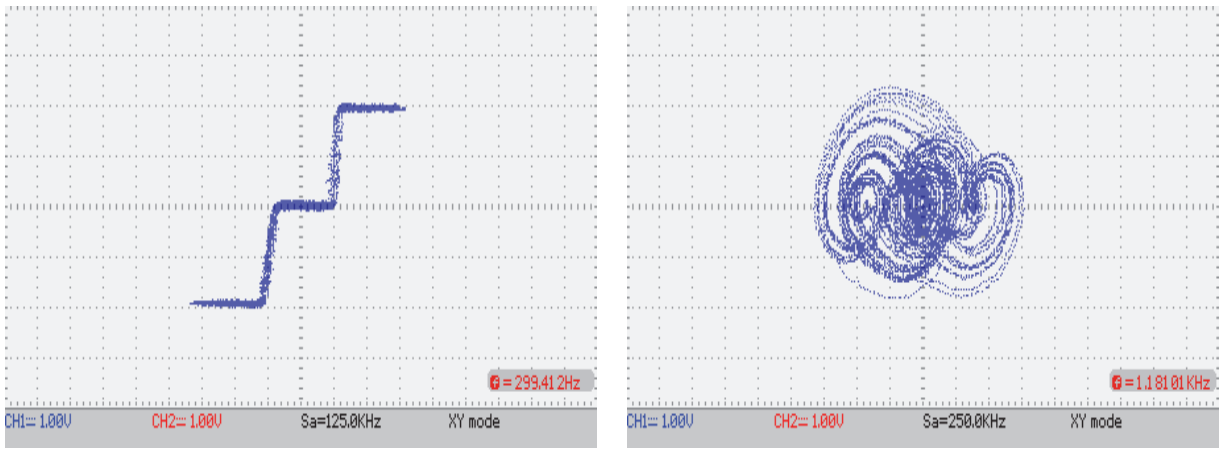

(a) Slopes: $k v=5$ and 10. Ch1:1V/Div, Ch2:1V/Div
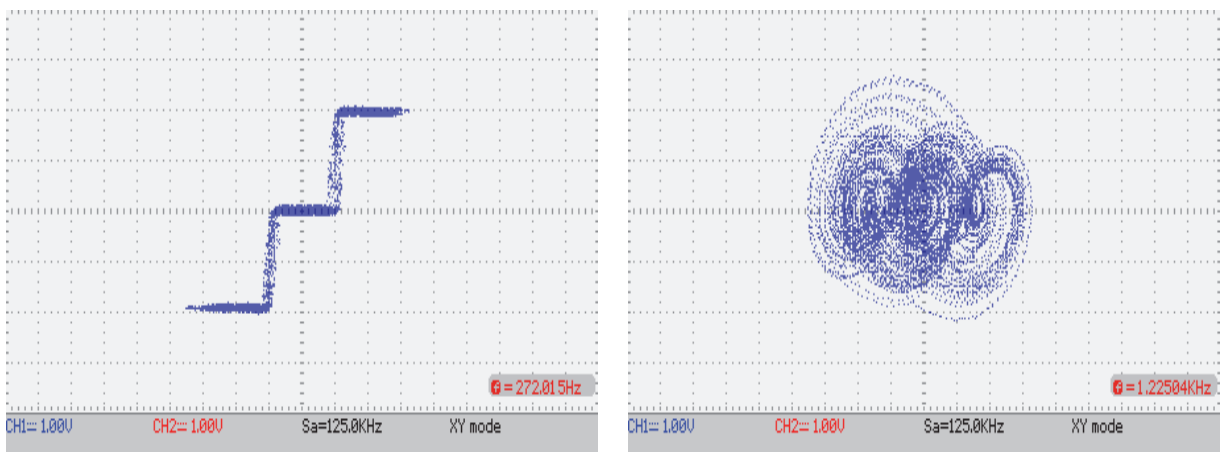

(b) Slopes: $k v=10$ and 20. Ch1:1V/Div, Ch2:1V/Div
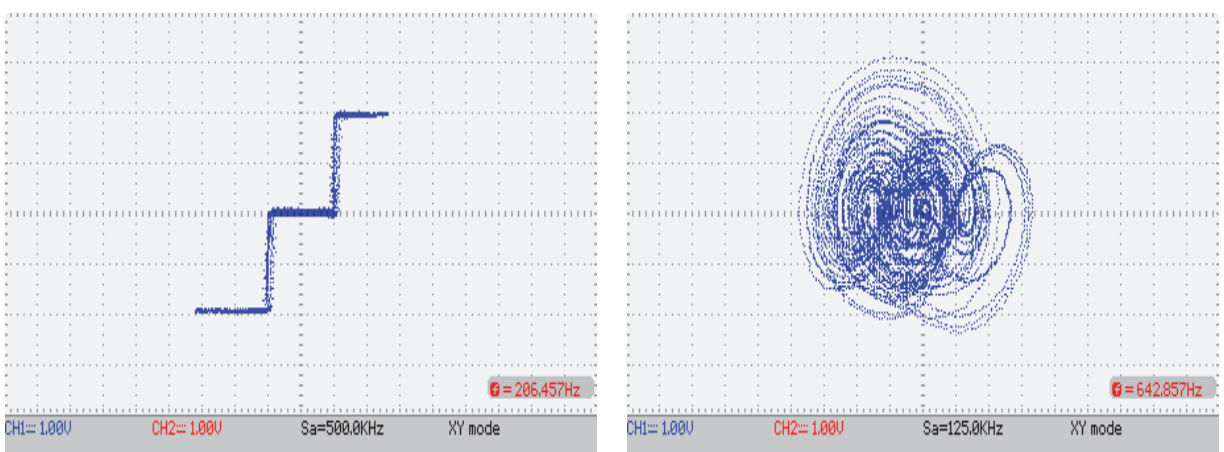

(c) Slopes: $k v=20$ and 50. Ch1:1V/Div, Ch2:1V/Div

FIGURE 6: Experimental verification of optimized 3 scrolls using irregular SNLFs with different slopes given in Table 3.

again using the slopes listed in Table 3 and Figure 1. Again, the circuit element values for realizing the three slopes are taken from Table 2. Figure 7 shows the experimental results for the irregular SNLF and the associated chaotic attractor showing 4 scrolls. As one can infer, according to Table 1, the first case for 4 scrolls uses the minimum slope value of 5 , which is also appreciated in the irregular SNLF shown in Figure 7(a).

\subsection{Optimized 5-Scroll Chaotic Oscillator with Different} Slopes. Generating 5 scrolls with optimized positive LE implies using four slopes, which are listed in Table 3. For those cases and by setting traditional values of $a=b=$ $c=d_{1}=0.7$, the positive LE is listed in Table 4, where their magnitudes are slightly more higher than those ones for 3 and 4 scrolls in Table 4. Again, positive LE increases by augmenting the number of scrolls from 4 to 5 .

The optimized positive LE is listed in Table 4, which is also higher than for generating 4 scrolls. The irregular SNLFs are realized again using the slopes listed in Table 3 and Figure 1. Four slopes are used because the SNLF shown in Figure 1(b) is increased. Once again, the circuit element values are taken from Table 2. Figure 8 shows the experimental results for the irregular SNLF and the associated chaotic attractor showing 5 scrolls.

In this section the irregular SNLFs was realized by modifying the values of slopes $k v$. The experiments confirmed the generation of 3,4 , and 5 scrolls that have an optimized positive LE, which was computed by applying $\mathrm{DE}$ algorithm in [22]. 

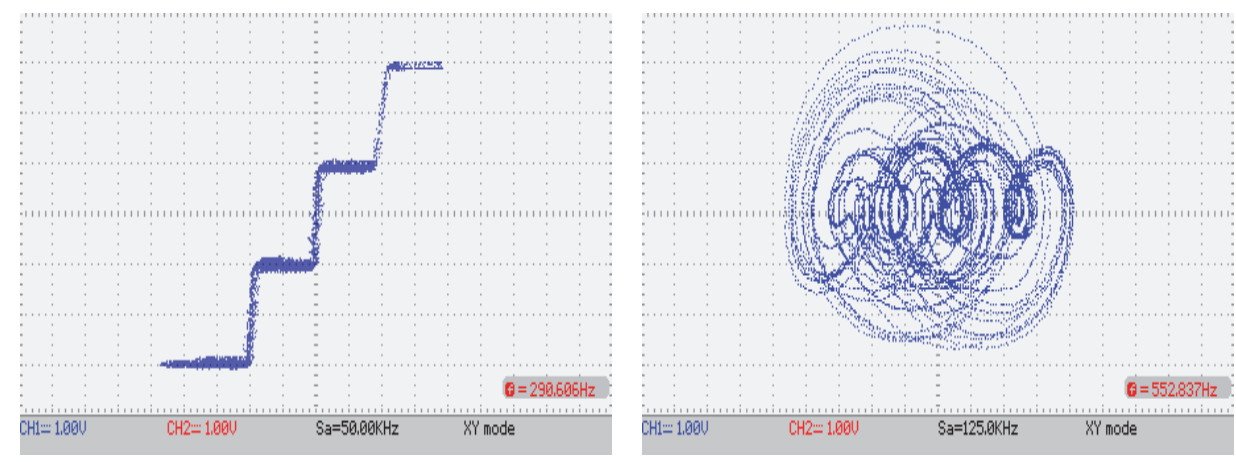

(a) Slopes: $k v=10,10$, and 5. Ch1:1V/Div, Ch2:1V/Div
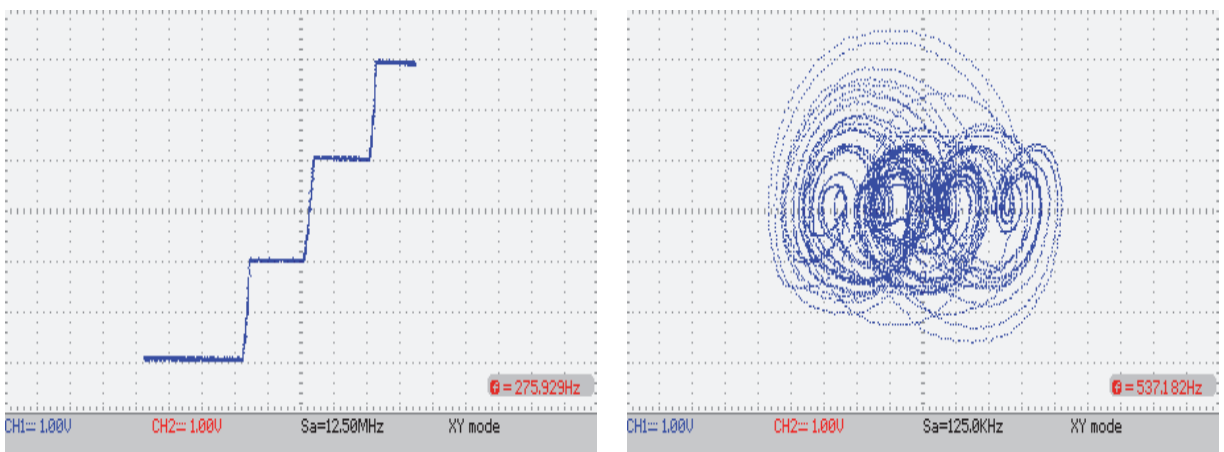

(b) Slopes: $k v=50,10$, and 20. Ch1:1V/Div, Ch2:1V/Div
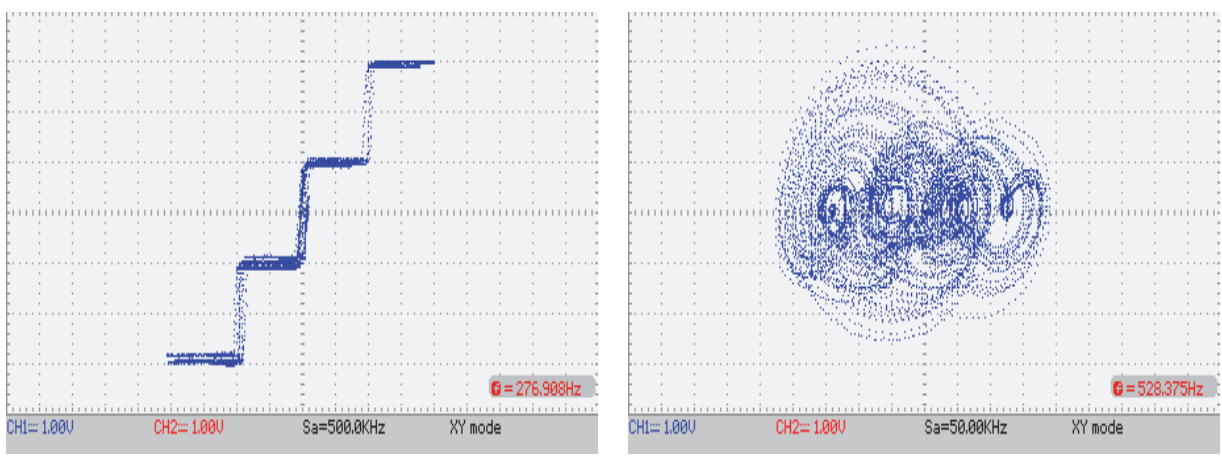

(c) Slopes: $k v=30,50$, and 70. Ch1:1V/Div, Ch2:1V/Div

FIGURE 7: Experimental verification of optimized 4 scrolls using irregular SNLFs with different slopes given in Table 3.

\section{Experimental Results by Varying the Delay of Slopes in SNLFs}

This section shows the experimental verification of optimized multiscroll chaotic oscillators by varying asymmetrically the delay $h$ (see Figure 1) of slopes $k v$, that is, the distance separating the center of slope with respect to the horizontal axis. Contrary to the previous section, the value of slopes $k v$ is keept as a constant for all cases, for example, $k v=10$. Also, the saturation levels $k$ are the same.

The physical realization of irregular SNLFs using commercially available OpAmps requires multiple voltage dividers to get the required voltages $E_{i}$ in Figure 3 . Table 5 lists the values of $h$ for each slope, according to the number of scrolls to be generated.
5.1. Optimized 2-Scroll Chaotic Oscillator with Different Delays. Table 6 lists the positive LEs of three cases from Table 5 by setting traditional values of $a=b=c=d_{1}=0.7$. In this case, only case 1 shows chaotic regime because cases 2 and 3 do not have a positive LE. However, after applying the optimization algorithm from [22], all cases have a positive LE, as listed in Table 6.

The experimental realization is performed by using system's parameters listed in Table 6 and the irregular SNLF with delays $h$ listed in Table 5 . In case $1, h=-0.5 \mathrm{~V}$ for $E_{i}$ in Figure 3, so that the saturation region $k$ on the right is wider that the left one. This gives as a result a larger scroll in the region of larger saturation width, as shown in Figure 9(a). The center of slope $k v$ defined by delay $h$ is the connecting point of neighbouring scrolls, that is, at $-0.5 \mathrm{~V}$. The other 
TABLE 5: Proposed values for modifying the delay $h$.

\begin{tabular}{lccc}
\hline Scrolls & Case 1 & Case 2 & Case 3 \\
\hline 2 & {$[-0.5]$} & {$[1]$} & {$[1.5]$} \\
3 & {$[-1,1.5]$} & {$[-2,1]$} & {$[-1.5,2]$} \\
4 & {$[-2,0.5,2]$} & {$[-2.5,0,1.5]$} & {$[-1.5,0,2]$} \\
5 & {$[-3,-1,1.3,2.5]$} & {$[-3,-1.5,0.5,2.5]$} & {$[-2.5,-1,1,2.5]$} \\
\hline
\end{tabular}
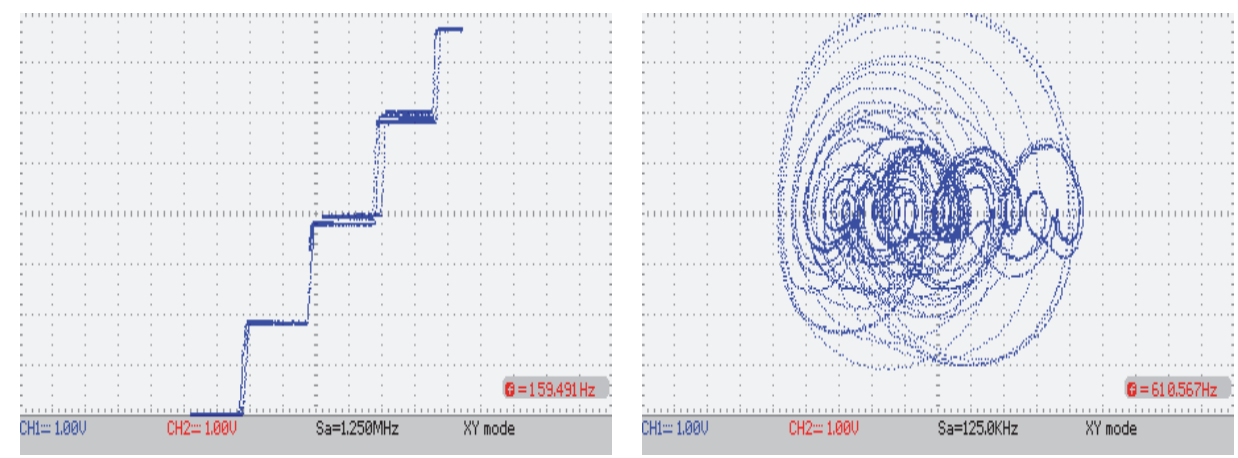

(a) Slopes: $k v=10,10,20$, and 20. Ch1:1V/Div, Ch2:1V/Div
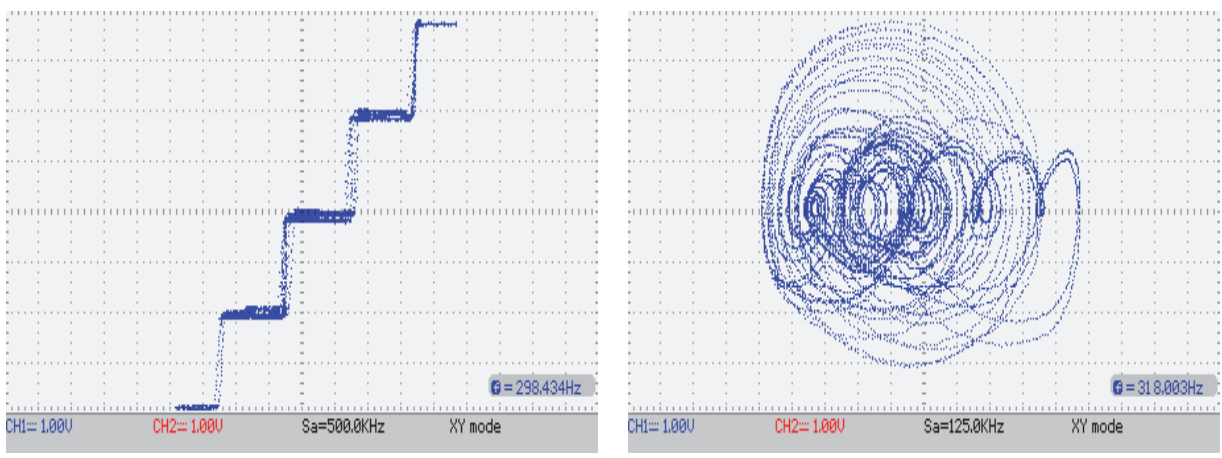

(b) Slopes: $k v=10,30,30$, and 10. Ch1:1V/Div, Ch2:1V/Div
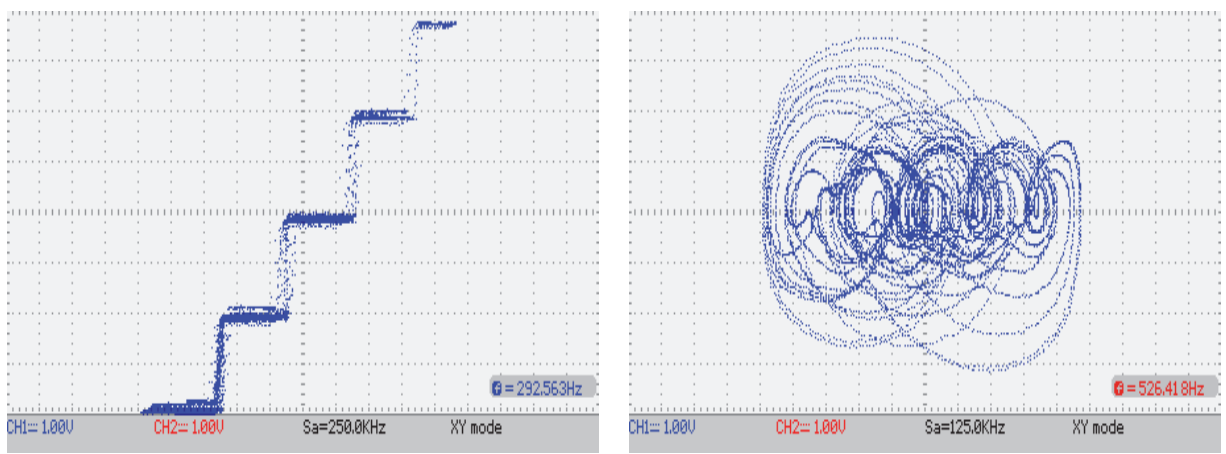

(c) Slopes: $k v=10,100,10$, and 100. Ch1:1V/Div, Ch2:1V/Div

FIGURE 8: Experimental verification of optimized 5 scrolls using irregular SNLFs with different slopes given in Table 3.

two cases are shown in Figures $9(\mathrm{~b})$ and $9(\mathrm{c})$, respectively.

5.2. Optimized 3-Scroll Chaotic Oscillator with Different Delays. Table 6 lists the positive LE for generating 3 scrolls and by setting traditional values of $a=b=c=d_{1}=0.7$.
All cases have a positive LE. After applying the optimization algorithm from [22], the value of positive LE is increased as listed in Table 6. Again, as for the previous section, the chaotic complexity is being increased by augmenting the number of scrolls. From Table 5, the three cases for obtaining 3 scrolls and by varying the delay $h$ are shown in Figure 10 . 
TABLE 6: Positive LEs for the optimized multiscrolls chaotic oscillators with different delays $h$ as the cases listed in Table 5.

\begin{tabular}{cccccccc}
\hline Case & Scroll number & $\begin{array}{c}\text { Positive LE without } \\
\text { optimization }\end{array}$ & $a$ & $b$ & $c$ & $d_{1}$ & $\begin{array}{c}\text { Positive LE with optimization } \\
\text { and irregular SNLF }\end{array}$ \\
\hline \multirow{3}{*}{1} & 2 & 0.0263 & 0.495 & 0.620 & 0.428 & 1.000 & 0.2671 \\
& 3 & 0.2166 & 0.747 & 0.618 & 0.490 & 0.722 & 0.3900 \\
& 4 & 0.2283 & 1.000 & 0.642 & 0.514 & 1.000 & 0.4318 \\
& 5 & 0.2865 & 0.851 & 0.693 & 0.292 & 0.731 & 0.4143 \\
\hline & 2 & -0.0182 & 0.628 & 0.486 & 1.000 & 1.000 & 0.3385 \\
& 3 & 0.0250 & 0.625 & 0.585 & 0.367 & 0.997 & 0.4144 \\
& 4 & -0.0324 & 0.998 & 0.735 & 0.504 & 0.774 & 0.4612 \\
& 5 & 0.0259 & 0.851 & 0.693 & 0.292 & 0.731 & 0.4143 \\
& 2 & 0 & 0.553 & 0.919 & 0.607 & 1.000 & 0.1688 \\
& 3 & 0.2221 & 1.000 & 0.853 & 0.546 & 1.000 & 0.4238 \\
& 4 & 0.0261 & 0.975 & 0.903 & 0.319 & 1.000 & 0.4466 \\
\end{tabular}
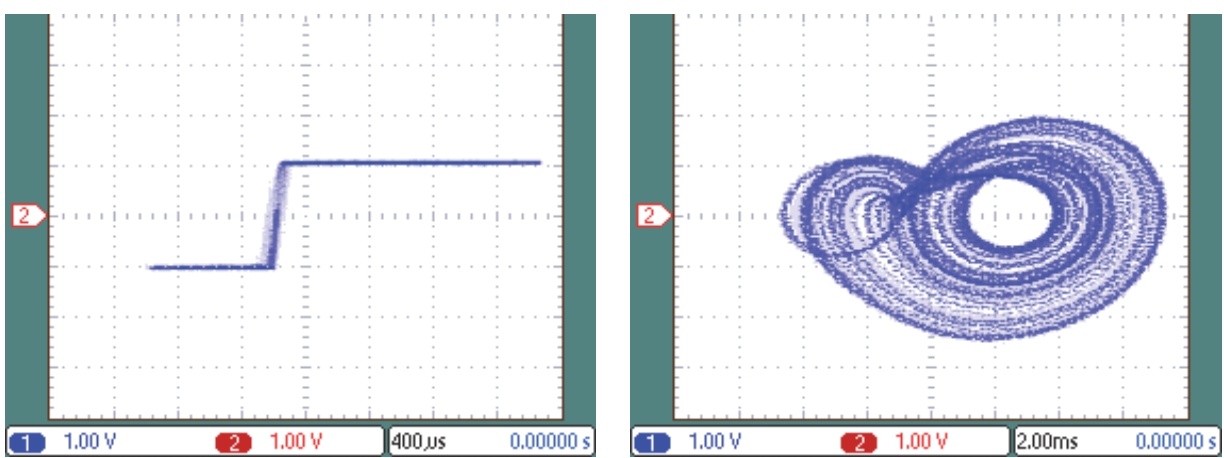

(a) Case 1: $h=-0.5$. Ch1:1V/Div, Ch2:1V/Div
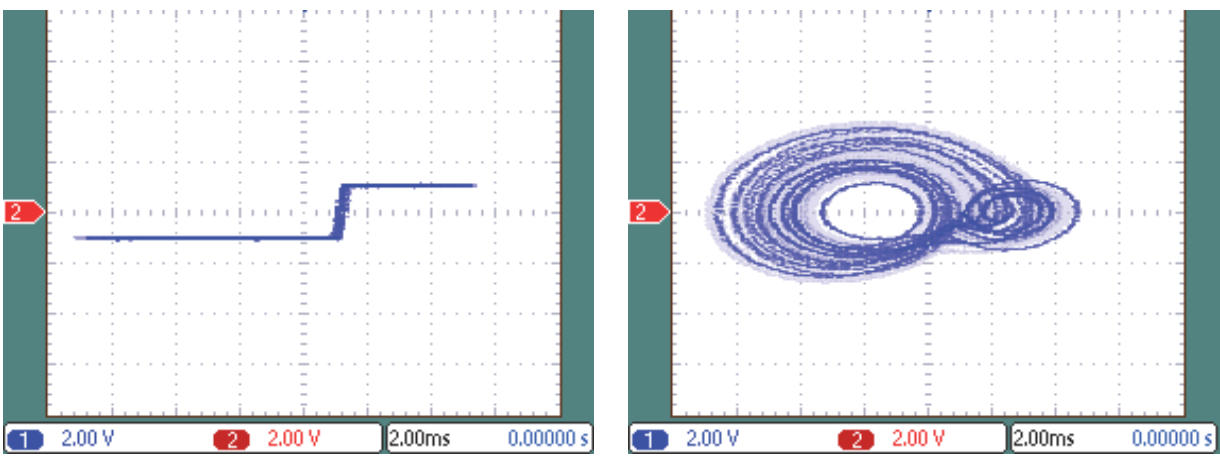

(b) Case 2: $h=1$. Ch1:2V/Div, Ch2:2V/Div
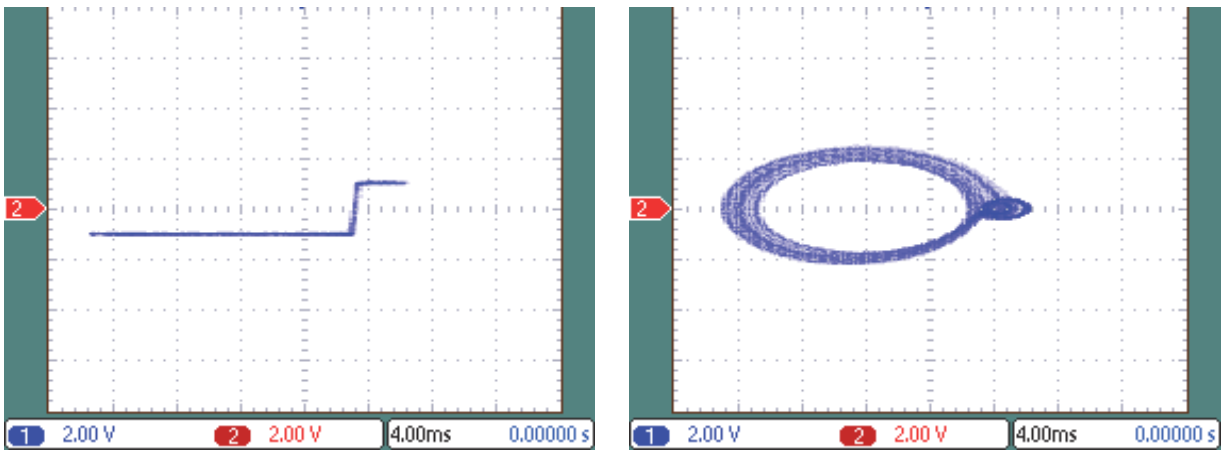

(c) Case 3: $h=1.5$. Ch1:2V/Div, Ch2:2V/Div

FIgURE 9: Experimental verification of optimized 2 scrolls using irregular SNLFs with different delays given in Table 5. 

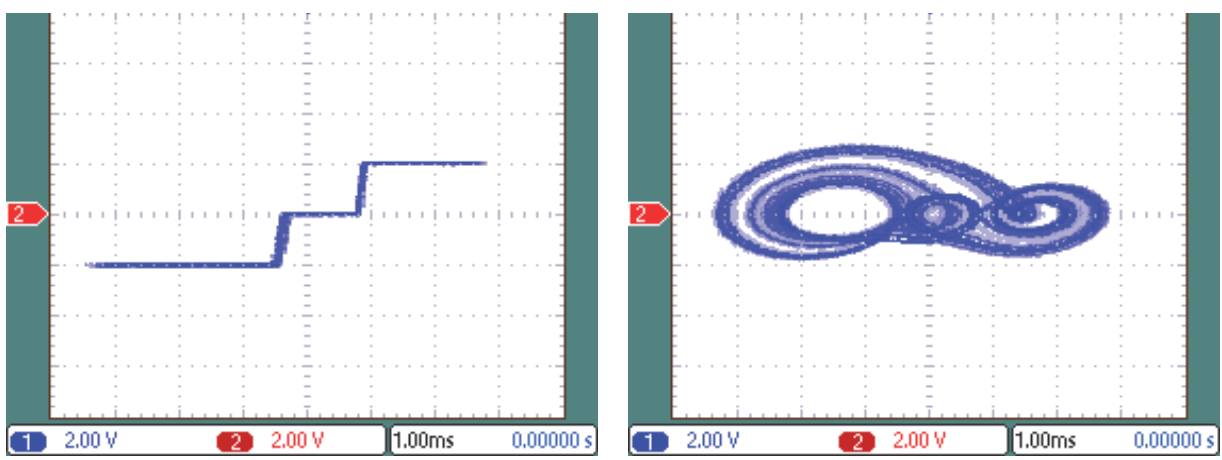

(a) Case 1: $h=-1$ and 1.5. Ch1:2V/Div, Ch2:2V/Div
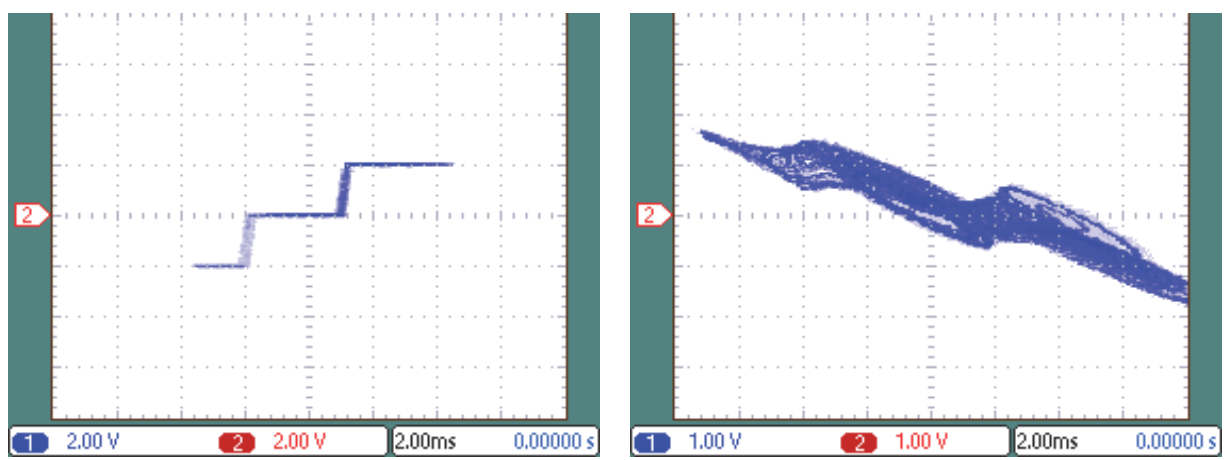

(b) Case 2: $h=-2$ and 1. Plotting state variables $x$ versus $z$, instead of $x$ versus $y$. On the left, Ch1:2V/Div, Ch2:2V/Div; on the right Ch1:1V/Div, Ch2:1V/Div
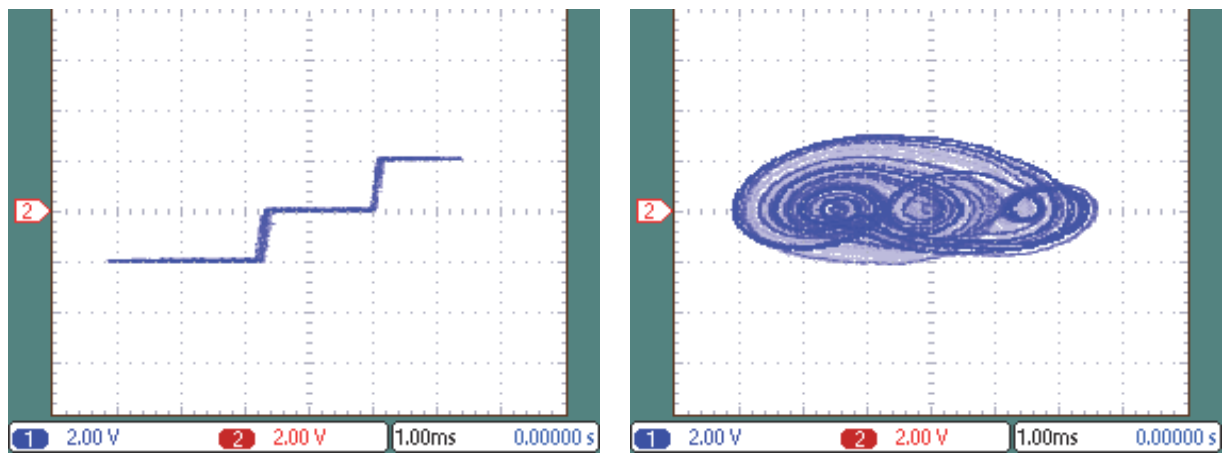

(c) Case 3: $h=-1.5$ and 2. Ch1:2V/Div, Ch2:2V/Div

FIGURE 10: Experimental verification of optimized 3 scrolls using irregular SNLFs with different delays given in Table 5.

5.3. Optimized 4-Scroll Chaotic Oscillator with Different Delays. The three cases for getting 4 scrolls and by setting traditional values of $a=b=c=d_{1}=0.7$ have the positive $\mathrm{LE}$ listed in Table 6. Not all the cases have a positive LE; however, after applying the optimization DE algorithm from [22], all cases have a greater positive LE than for 3 scrolls, as listed in Table 6, thus confirming again that the chaotic complexity is being increased by augmenting the number of scrolls. From Table 5, the three cases for obtaining 4 scrolls and by varying the delay $h$ are shown in Figure 11.

5.4. Optimized 5-Scroll Chaotic Oscillator with Different Delays. Finally, the three cases for getting 5 scrolls and by setting traditional values of $a=b=c=d_{1}=0.7$ have the positive LE listed in Table 6 . All the cases have a positive LE, and they are optimized by applying the optimization DE algorithm [22], where only case 3 has a positive LE greater than for generating 4 scrolls, as listed in Table 6. Anyway, the chaotic complexity is being increased by augmenting the number of scrolls. From Table 5, the three cases for attaining 5 scrolls and by varying the delay $h$ are shown in Figure 12 .

\section{Synchronization between Optimized and Nonoptimized Multiscroll Chaotic Attractors}

In this section the synchronization between optimized and nonoptimized multiscroll chaotic attractors is shown by 

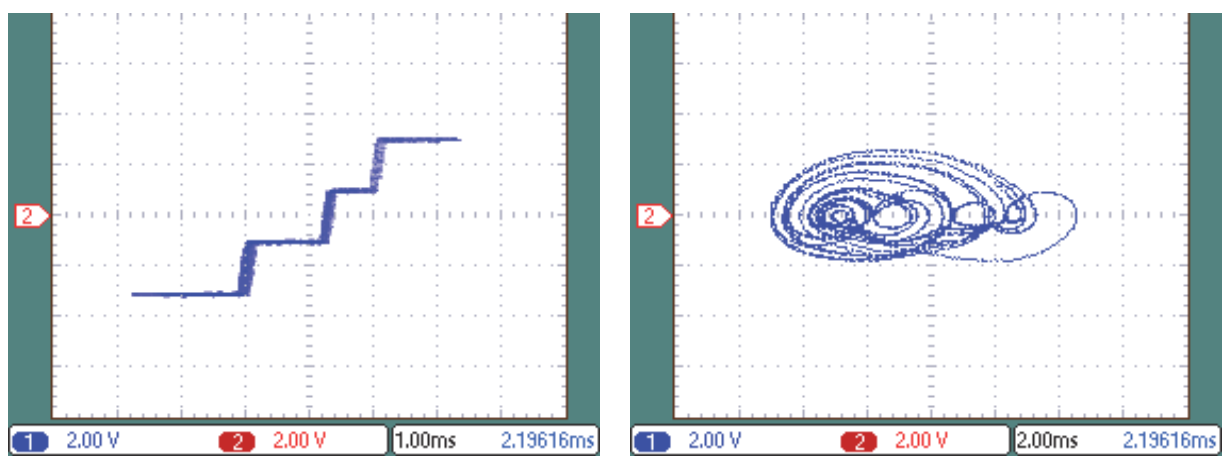

(a) Case 1: $h=-2,0.5$ and 2. Ch1:2V/Div, Ch2:2V/Div
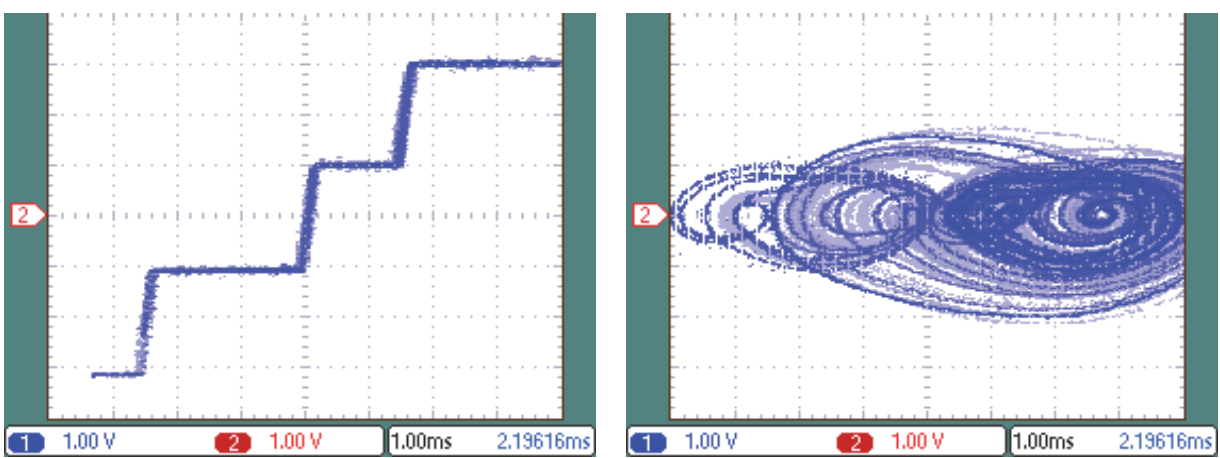

(b) Case 2: $h=-2.5,0$ and 1.5. Ch1:1V/Div, Ch2:1V/Div
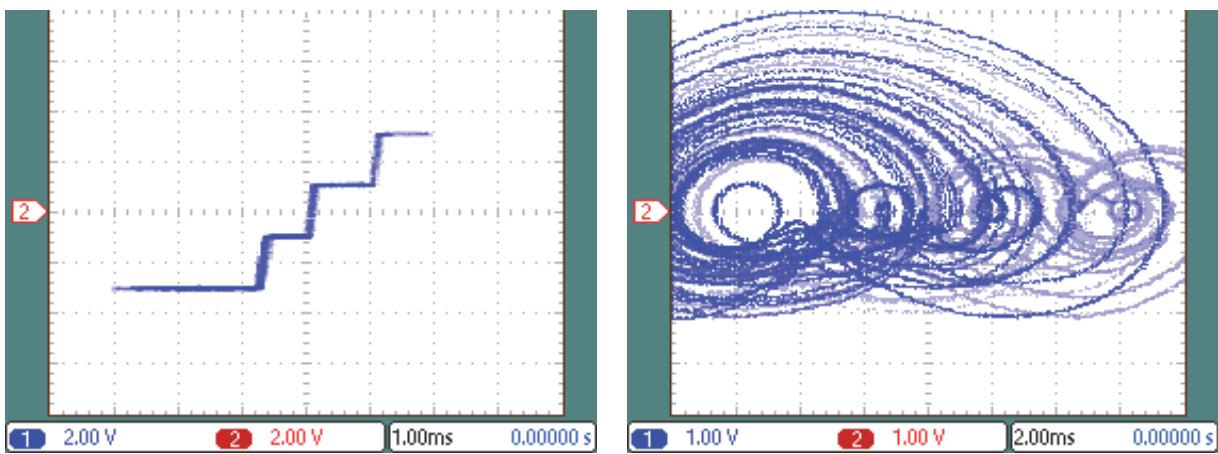

(c) Case 3: $h=-1.5,0$ and 2. On the left Ch1:2V/Div, Ch2:2V/Div, and on the right Ch1:1V/Div, Ch2:1V/Div

FIGURE 11: Experimental verification of optimized 4 scrolls using irregular SNLFs with different delays given in Table 5.

applying the proposed approach given in [39], that is, generalized Hamiltonian forms. Besides, it is demonstrated that, by using an optimized 4-scroll chaotic oscillator as master system, the slave system that is not optimized can show a similar behavior. This is an important result since it is not necessary to optimize each chaotic oscillator independently.

Then, let us take the multiscroll chaotic system (1) as the master system as follows:

$$
\begin{aligned}
& \dot{x}=y, \\
& \dot{y}=z, \\
& \dot{z}=-a x-b y-c z+d_{1} f(x ; q) .
\end{aligned}
$$

By using a Hamilton energy function defined by $H(x)=$ $1 / 2\left[a x^{2}+b y^{2}+z^{2}\right]$, we can obtain the slave system defined by

$$
\begin{aligned}
& \dot{\hat{x}}=\widehat{y}+k_{1}(x-\widehat{x}), \\
& \dot{\hat{y}}=\widehat{z}+k_{2}(x-\widehat{x}), \\
& \dot{\bar{z}}=-a \hat{x}-b \hat{y}-c \widehat{z}+d_{1} \widehat{f}(x ; \widehat{q})+k_{3}(x-\widehat{x}),
\end{aligned}
$$

which synchronizes with master system (4) when the synchronization error $x-\hat{x}$ tends to zero by selecting appropriate synchronization gains $k_{1}, k_{2}, k_{3}$. The synchronization gains are selected as explained in detail in [39]. 

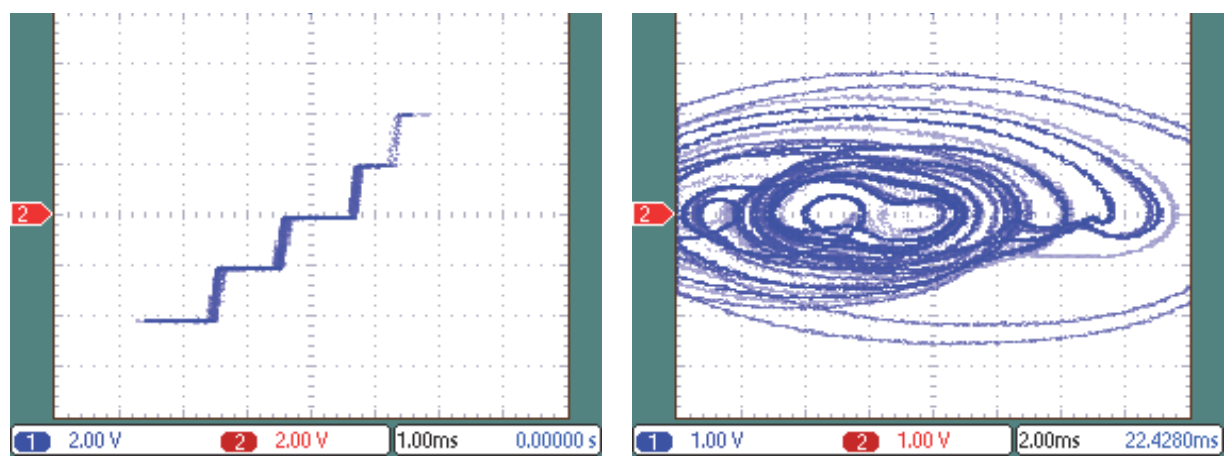

(a) Case 1: $h=-3,-1,1.3$ and 2.5. On the left Ch1:2V/Div, Ch2:2V/Div, and on the right Ch1:1V/Div, Ch2:1V/Div
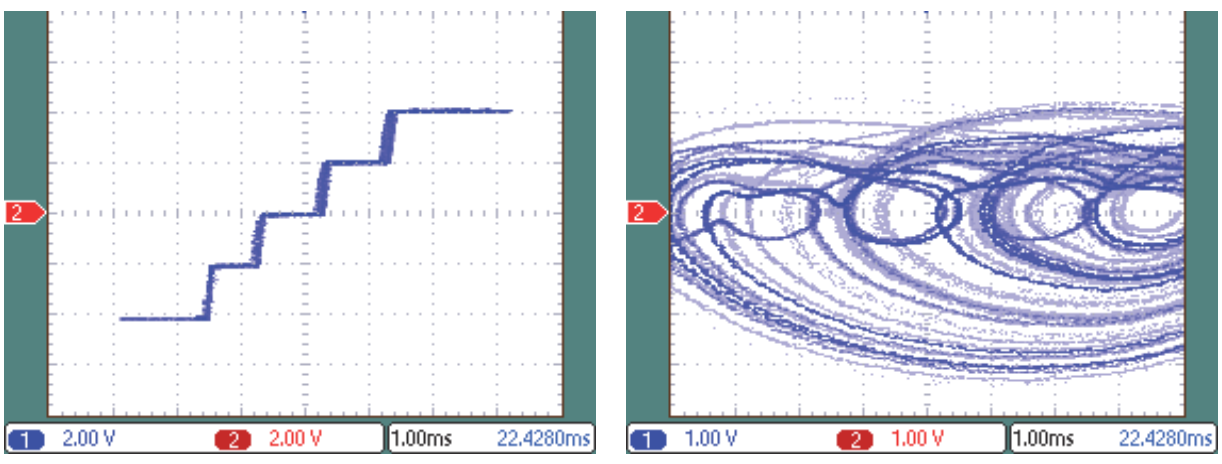

(b) Case 2: $h=-3,-1.5,0.5$ and 2.5. On the left Ch1:2V/Div, Ch2:2V/Div, and on the right Ch1:1V/Div, Ch2:1V/Div
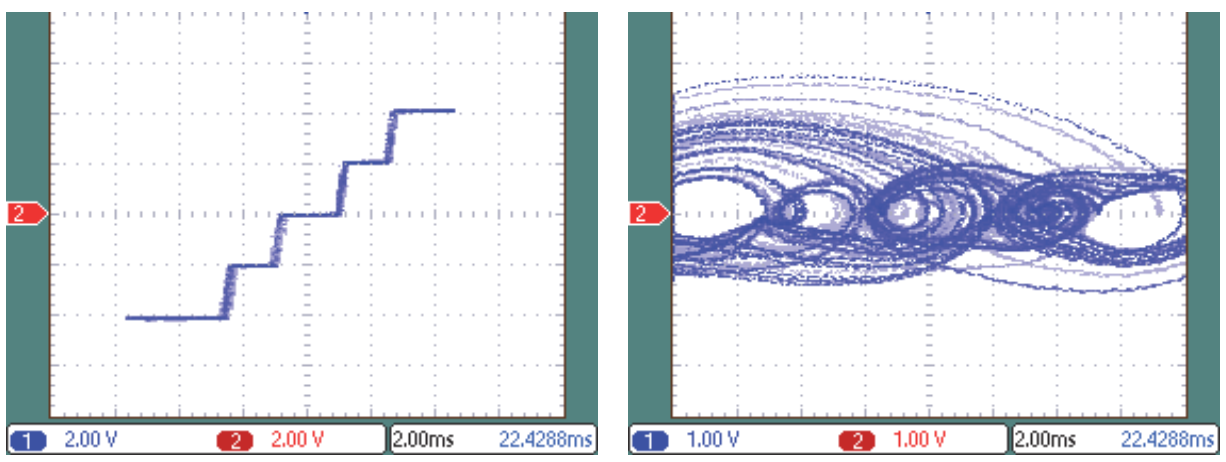

(c) Case 3: $h=-2.5,-1,1$ and 2.5. On the left Ch1:2V/Div, Ch2:2V/Div, and on the right Ch1:1V/Div, Ch2:1V/Div

FIGURE 12: Experimental verification of optimized 5 scrolls using irregular SNLFs with different delays given in Table 5.

6.1. Synchronization of Nonoptimized 4-Scroll with Case 1 in Table 4: Different Slopes. By using different slopes for SNLF (irregular) as given in case 1, Table 4, the resulting 4-scroll chaotic attractor can be synchronized with another 4-scroll chaotic attractor that preserves traditional values for system's parameters as well as a regular SNLF. For master system, we use the slopes $=10,10,5$ (case 1, Table 4) for irregular SNLF, system's parameters $a=1.0, b=0.709, c=0.290, d_{1}=1.0$, synchronization gains $k_{1}=4, k_{2}=20, k_{3}=45$, and initial conditions $x(0)=1, y(0)=1, z(0)=0.01$. On the other hand, for slave system traditional values $a=0.7, b=0.7, c=$ $0.7, d_{1}=0.7$ are considered; also a symmetrical SNLF with slopes $=200$ are used, and $\widehat{x}(0)=0.1, \widehat{y}(0)=0.1, \widehat{z}(0)=0.1$. Figure 13 shows the simulation results of synchronization. In Figure 13(a) the phase portraits for master system and slave system without and with synchronization are given. Figures 13(b) and 13(c) show the $x-z$ and $y-z$ planes for the same 4 -scroll attractor. Synchronization error is represented as a straight line in phase planes $x-\widehat{x}, y-\widehat{y}$, and $z-\widehat{z}$ as shown in Figure 13(d).

6.2. Synchronization of Nonoptimized 4-Scroll with Case 1 in Table 6: Different Delays. Similar to previous section, by using different delays for slopes in SNLF (irregular) as given in case 1 , Table 6 , the resulting 4 -scroll chaotic attractor can be synchronized with another 4-scroll chaotic attractor that preserves traditional values for system's parameters as well as a regular SNLF. For master system, we use different delays $h=-2,0.5,2$, slope $=10$ (case 1 , Table 6) for irregular SNLF, system's parameters $a=1.0, b=0.642, c=0.514, d_{1}=1.0$, 

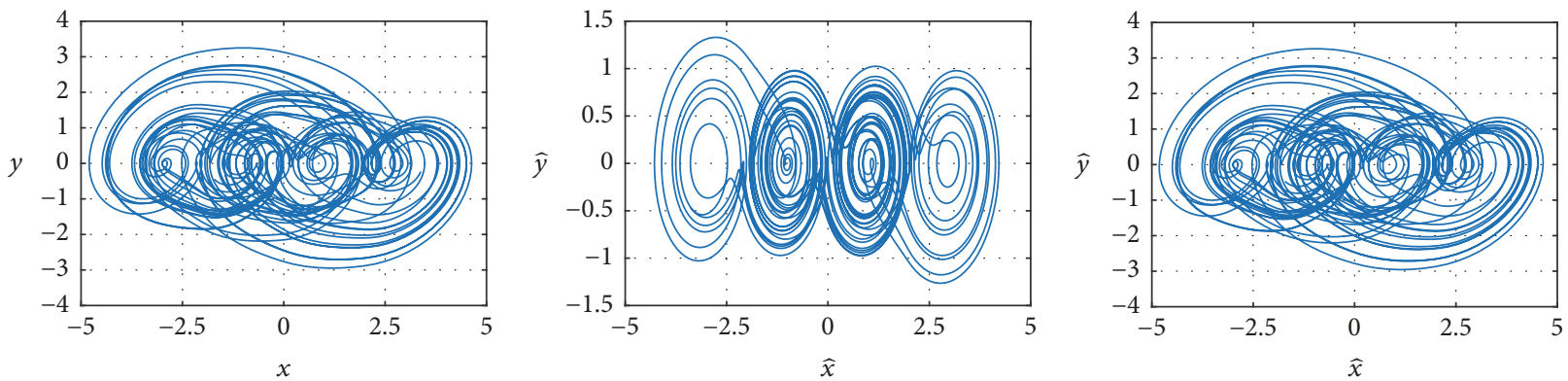

(a) Optimized 4-scroll chaotic system, typical 4-scroll chaotic system without optimization, and typical 4-scroll chaotic system after synchronization $(x-y$ plane $)$
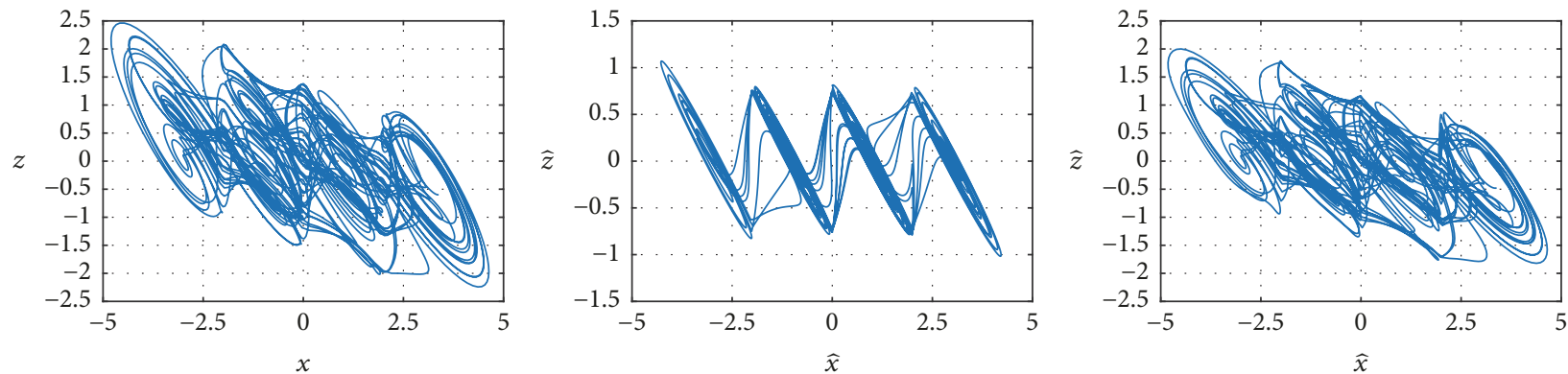

(b) Optimized 4-scroll chaotic system, typical 4-scroll chaotic system without optimization, and typical 4-scroll chaotic system after synchronization $(x-z$ plane $)$
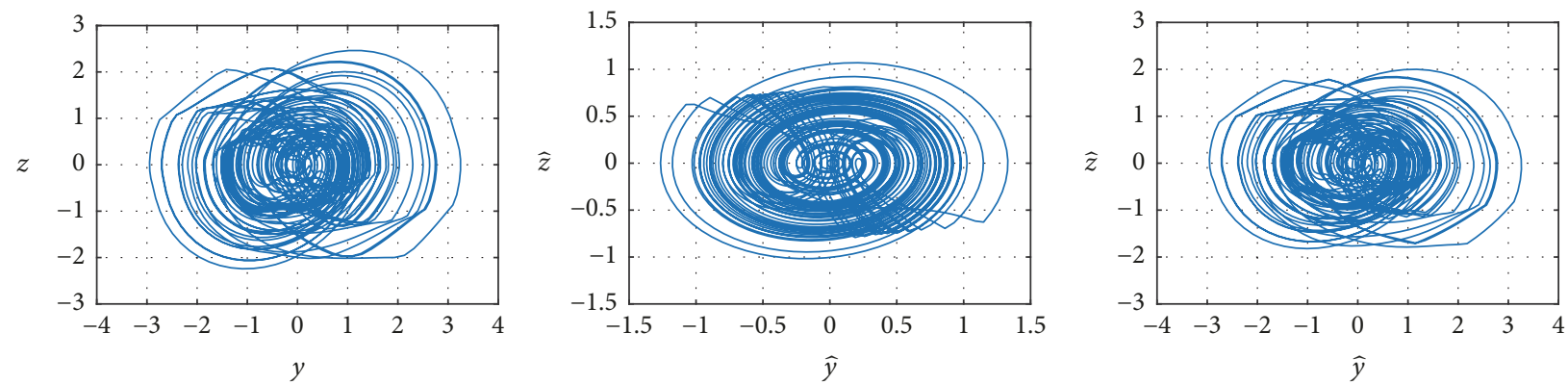

(c) Optimized 4-scroll chaotic system, typical 4-scroll chaotic system without optimization, and typical 4-scroll chaotic system after synchronization $(y-z$ plane $)$
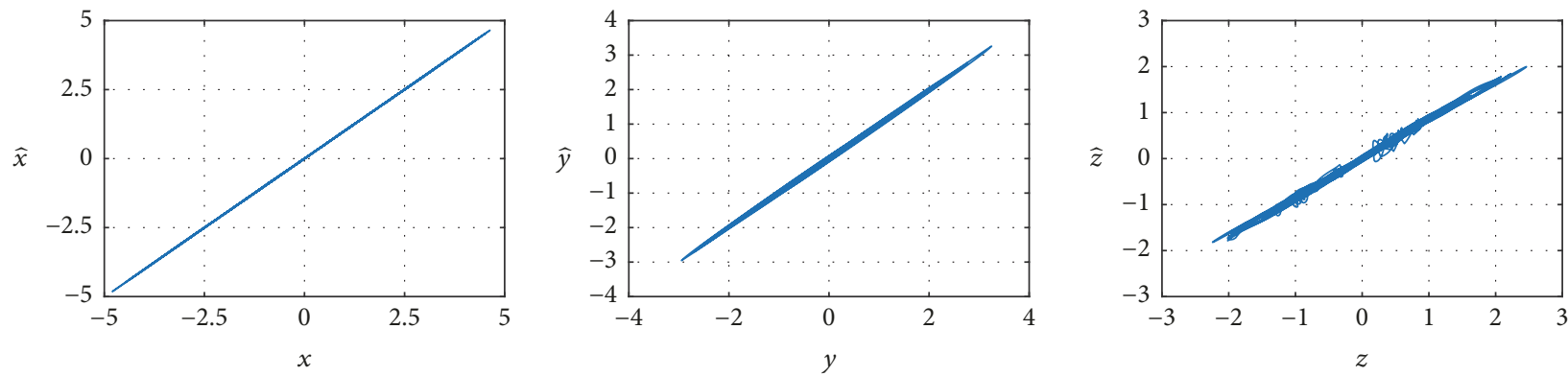

(d) Synchronization error for optimized and nonoptimized 4-scroll chaotic system

FIGURE 13: Synchronization results between optimized and nonoptimized chaotic oscillators with different slopes.

synchronization gains $k 1=4, k 2=20, k 3=45$, and initial conditions ic $=2.99,-0.03,0.1$. On the other hand, for slave system traditional values $a=0.7, b=0.7, c=0.7$, $d_{1}=0.7$ are considered; also a symmetrical SNLF with slopes $=200$ and delays $h=-2,0,2$ are used, ic $=2.9,0.01,0.01$. Figure 14 shows the simulation results of synchronization.
In Figure 14(a) the phase portraits for master system and slave system without and with synchronization are given. Synchronization error between $x-\widehat{x}, y-\widehat{y}$, and $z-\widehat{z}$ is given in Figure 14(b).

Next, Figure 15 shows the transient response for synchronization. In both cases, the numerical simulations confirm an 

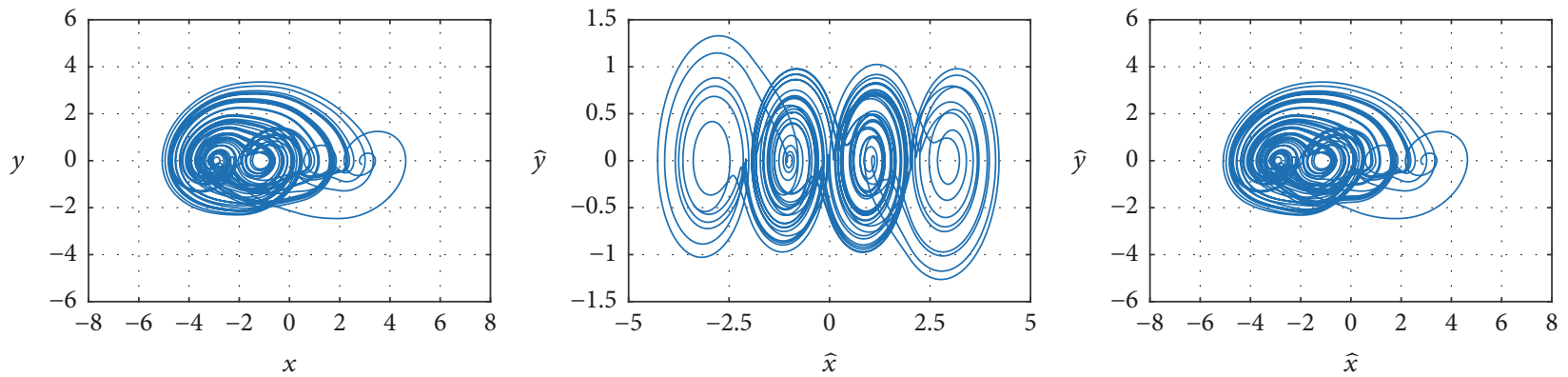

(a) Optimized 4-scroll chaotic system, typical 4-scroll chaotic system without optimization, and typical 4-scroll chaotic system after synchronization $(x-y$ plane $)$
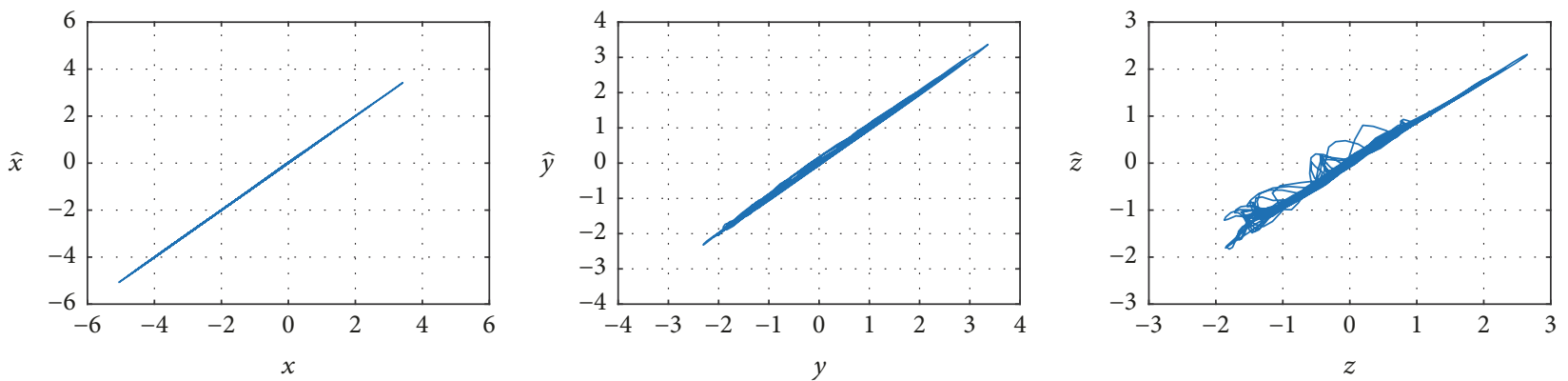

(b) Synchronization error for optimized and nonoptimized 4-scroll chaotic system

FIGURE 14: Synchronization results between optimized and nonoptimized chaotic oscillators with different delays of the slopes.
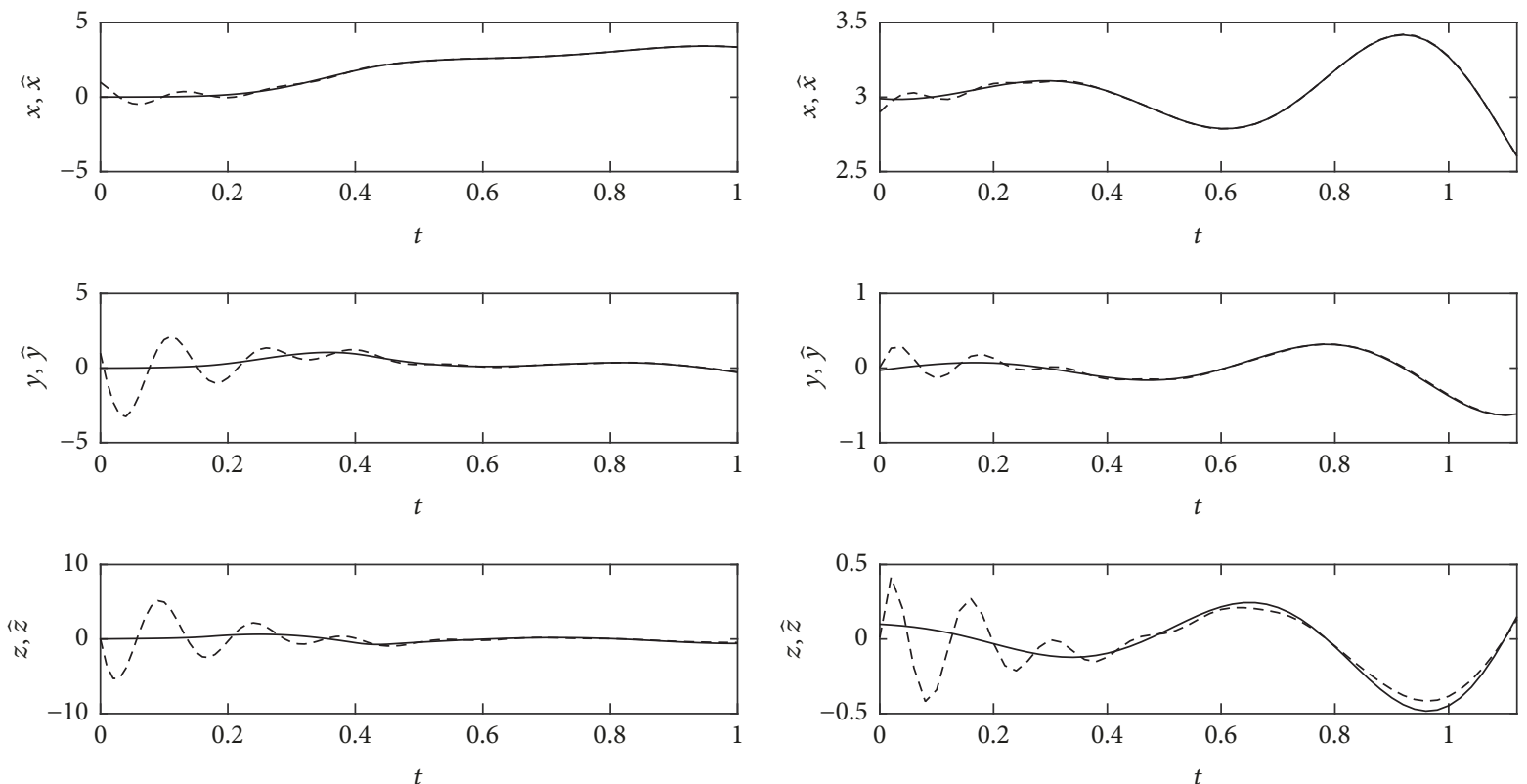

Figure 15: Transient response for both cases in Figures 13 and 14, respectively.

adequate synchronization between optimized and nonoptimized multiscroll chaotic oscillators.

As a general result, the experimental realizations of optimized multiscroll chaotic oscillators confirmed that the irregular SNLF provides a suitable mechanism to obtain a positive LE with a higher magnitude than without optimization as shown in Tables 4 and 6 . In addition, the irregular SNLF proposed in this paper leads to getting a high value of the positive LE when compared to other approaches also using evolutionary algorithms to maximize the positive LE as shown in Table 7. The main characteristics, such as maximum value of the positive LE, experimental realization, and synchronization, of this work are given in Table 7. 
TABLE 7: Comparison of the main results of this work with other approaches.

\begin{tabular}{lccc}
\hline Characteristic & Ref [22] & Ref [19] & This work \\
\hline $\begin{array}{l}\text { Multiscrolls } \\
\text { Optimization algorithm }\end{array}$ & $\checkmark(2$ to 9 scrolls $)$ & $\checkmark(2$ to 6 scrolls) & $\checkmark(2$ to 5 scrolls) \\
Type of SNLF & $\begin{array}{l}\text { Regular } \\
\text { (symmetrical) }\end{array}$ & $\checkmark$ (DE) & $\checkmark$ (DE) \\
Maximum value of the positive & 0.4416 & (symmetrical) & Irregular (asymmetrical) \\
LE after optimization (5 scrolls) & - & 0.2711 & 0.7112 (case 3 in Table 4$)$ \\
$\begin{array}{l}\text { Experimental verification } \\
\text { Synchronization scheme }\end{array}$ & - & - & $\checkmark(2$ to 5 scrolls) \\
\end{tabular}

\section{Conclusions}

It has been shown the experimental realization of optimized multiscrolls chaotic attractors. More specifically, 2 to 5 scrolls were obtained by using irregular SNLFs. Two cases were considered: variation of the slopes of SNLF and variation of the delays of slopes. Both cases led us to implement irregular SNLFs and, by applying an optimization evolutionary algorithm, the positive LE was maximized. From the experimental results, it can be appreciated that the higher value of positive Lyapunov exponents is obtained by varying the slopes of SNLF. Also, all the experiments listed in the last two sections confirmed that the value of positive LE increases by augmenting the number of scrolls. On the other hand, the synchronization between optimized and nonoptimized chaotic attractors was demonstrated. Further, this research could be useful to propose engineering applications based on chaos, for example, secure communications, since the obtained results have been performed at experimental circuit level.

\section{Conflicts of Interest}

The authors declare that they have no conflicts of interest.

\section{Acknowledgments}

This work has been partially supported by CONACYT-SNI (Mexico). The authors thankfully acknowledge the computer resources, technical expertise, and support provided by the "Laboratorio Nacional de Supercómputo del Sureste de México (National Laboratory of High Performance Computing)," belonging to CONACYT Network of National Laboratories. J. M. Muñoz-Pacheco acknowledges CONACYT for the financial support (no. 258880: Proyecto Apoyado por el Fondo Sectorial de Investigación para la Educación).

\section{References}

[1] J. C. Sprott, "New chaotic regimes in the Lorenz and Chen systems," International Journal of Bifurcation and Chaos, vol. 25, no. 2, Article ID 1550033, 2015.

[2] T. Gotthans and J. Petržela, "New class of chaotic systems with circular equilibrium," Nonlinear Dynamics, vol. 81, no. 3, pp. 1143-1149, 2015.
[3] Z. Wang, J. Ma, Z. Chen, and Q. Zhang, "A new chaotic system with positive topological entropy," Entropy, vol. 17, no. 8, pp. 5561-5579, 2015.

[4] V.-T. Pham, C. Volos, S. Jafari, Z. Wei, and X. Wang, "Constructing a novel no-equilibrium chaotic system," International Journal of Bifurcation and Chaos, vol. 24, no. 5, Article ID 1450073, 2014.

[5] D. Chen, Z. Sun, X. Ma, and L. Chen, "Circuit implementation and model of a new multi-scroll chaotic system," International Journal of Circuit Theory and Applications, vol. 42, no. 4, pp. 407-424, 2014.

[6] P. Muthukumar, P. Balasubramaniam, and K. Ratnavelu, "Fast projective synchronization of fractional order chaotic and reverse chaotic systems with its application to an affine cipher using date of birth (DOB)," Nonlinear Dynamics, vol. 80, no. 4, pp. 1883-1897, 2015.

[7] Y. Xu, H. Wang, Y. Li, and B. Pei, "Image encryption based on synchronization of fractional chaotic systems," Communications in Nonlinear Science and Numerical Simulation, vol. 19, no. 10, pp. 3735-3744, 2014.

[8] X.-Y. Ji, S. Bai, Y. Guo, and H. Guo, "A new security solution to JPEG using hyper-chaotic system and modified zigzag scan coding," Communications in Nonlinear Science and Numerical Simulation, vol. 22, no. 1-3, pp. 321-333, 2015.

[9] H.-I. Hsiao and J. Lee, "Fingerprint image cryptography based on multiple chaotic systems," Signal Processing, vol. 113, pp. 169181, 2015.

[10] L. J. Ontanon-Garcia, E. Jimenez-Lopez, E. Campos-Canton, and M. Basin, "A family of hyperchaotic multi-scroll attractors in Rn," Applied Mathematics and Computation, vol. 233, pp. 522533, 2014.

[11] J. Ma, Z. Chen, Z. Wang, and Q. Zhang, "A four-wing hyperchaotic attractor generated from a $4 \mathrm{D}$ memristive system with a line equilibrium," Nonlinear Dynamics, vol. 81, no. 3, pp. 12751288, 2015.

[12] E. Zambrano-Serrano, E. Campos-Cantón, and J. M. MuñozPacheco, "Strange attractors generated by a fractional order switching system and its topological horseshoe," Nonlinear Dynamics, vol. 83, no. 3, pp. 1629-1641, 2016.

[13] S. Hassan Hosseinnia, R. L. Magin, and B. M. Vinagre, "Chaos in fractional and integer order NSG systems," Signal Processing, vol. 107, pp. 302-311, 2015.

[14] Y. Xu, R. Gu, H. Zhang, and D. Li, "Chaos in diffusionless lorenz system with a fractional order and its control," International Journal of Bifurcation and Chaos, vol. 22, no. 4, Article ID 1250088, 2012. 
[15] Y. Xu, H. Wang, D. Liu, and H. Huang, "Sliding mode control of a class of fractional chaotic systems in the presence of parameter perturbations," Journal of Vibration and Control, vol. 21, no. 3, pp. 435-448, 2015.

[16] D. Liu, W. Xu, and Y. Xu, "Noise-induced chaos in the elastic forced oscillators with real-power damping force," Nonlinear Dynamics, vol. 71, no. 3, pp. 457-467, 2013.

[17] Y. Xu, R. Gu, and H. Zhang, "Effects of random noise in a dynamical model of love," Chaos, Solitons \& Fractals, vol. 44, no. 7, pp. 490-497, 2011.

[18] Y. Xu, G. M. Mahmoud, W. Xu, and Y. Lei, "Suppressing chaos of a complex Duffing's system using a random phase," Chaos, Solitons \& Fractals, vol. 23, no. 1, pp. 265-273, 2005.

[19] L. G. De La Fraga and E. Tlelo-Cuautle, "Optimizing the maximum Lyapunov exponent and phase space portraits in multi-scroll chaotic oscillators," Nonlinear Dynamics, vol. 76, no. 2, pp. 1503-1515, 2014.

[20] F. L. Dubeibe and L. D. Bermúdez-Almanza, "Optimal conditions for the numerical calculation of the largest Lyapunov exponent for systems of ordinary differential equations," International Journal of Modern Physics C, vol. 25, no. 7, Article ID 1450024, 2014.

[21] M. Ciobanu, A. Ardelean, C. Cotoraci, and L. Mos, "Maximum lyapunov exponents evidencing chaos in neural activity," Journal of Computational and Theoretical Nanoscience, vol. 10, no. 11, pp. 2600-2603, 2013.

[22] V. H. Carbajal-Gomez, E. Tlelo-Cuautle, and F. V. Fernandez, "Optimizing the positive Lyapunov exponent in multiscroll chaotic oscillators with differential evolution algorithm," Applied Mathematics and Computation, vol. 219, no. 15, pp. 8163-8168, 2013.

[23] C. Sanchez-Lopez, "Automatic synthesis of chaotic attractors," Applied Mathematics and Computation, vol. 217, no. 9, pp. 43504358, 2011.

[24] G. Ablay, "Novel chaotic delay systems and electronic circuit solutions," Nonlinear Dynamics, vol. 81, no. 4, pp. 1795-1804, 2015.

[25] M. Shahzad, V.-T. Pham, M. A. Ahmad, S. Jafari, and F. Hadaeghi, "Synchronization and circuit design of a chaotic system with coexisting hidden attractors," The European Physical Journal Special Topics, vol. 224, no. 8, pp. 1637-1652, 2015.

[26] J. Ma, X. Y. Wu, R. T. Chu, and L. Zhang, "Selection of multiscroll attractors in Jerk circuits and their verification using Pspice," Nonlinear Dynamics, vol. 76, no. 4, pp. 1951-1962, 2014.

[27] J. M. Muñoz-Pacheco, E. Tlelo-Cuautle, I. Toxqui-Toxqui, C. Sánchez-López, and R. Trejo-Guerra, "Frequency limitations in generating multi-scroll chaotic attractors using CFOAs," International Journal of Electronics, vol. 101, no. 11, pp. 1559-1569, 2014.

[28] Y. Ma, Y. Li, and X. Jiang, "Simulation and circuit implementation of 12-scroll chaotic system," Chaos, Solitons \& Fractals, vol. 75, pp. 127-133, 2015.

[29] J. B. Dingwell, Lyapunov Exponents. Wiley Encyclopedia of Biomedical Engineering, John Wiley \& Sons, 2006.

[30] A. Wolf, J. B. Swift, H. L. Swinney, and J. A. Vastano, "Determining Lyapunov exponents from a time series," Physica D: Nonlinear Phenomena, vol. 16, no. 3, pp. 285-317, 1985.

[31] R. Hegger, H. Kantz, and T. Schreiber, "Practical implementation of nonlinear time series methods: the TISEAN package," Chaos: An Interdisciplinary Journal of Nonlinear Science, vol. 9, no. 2, pp. 413-435, 1999.
[32] N. Look, C. J. Arellano, A. M. Grabowski, W. J. McDermott, R. Kram, and E. Bradley, "Dynamic stability of running: The effects of speed and leg amputations on the maximal lyapunov exponent," Chaos: An Interdisciplinary Journal of Nonlinear Science, vol. 23, no. 4, Article ID 043131, 2013.

[33] A. Sarkar, A. Narváez, and J. Harting, "Quantification of the performance of chaotic micromixers on the basis of finite time Lyapunov exponents," Microfluidics and Nanofluidics, vol. 13, no. 1, pp. 19-27, 2012.

[34] H. Liu, A. Abraham, and M. Clerc, "Chaotic dynamic characteristics in swarm intelligence," Applied Soft Computing, vol. 7, no. 3, pp. 1019-1026, 2007.

[35] D. Yang, Z. Liu, and J. Zhou, "Chaos optimization algorithms based on chaotic maps with different probability distribution and search speed for global optimization," Communications in Nonlinear Science and Numerical Simulation, vol. 19, no. 4, pp. 1229-1246, 2014.

[36] X. Ai, K. Sun, S. He, and H. Wang, "Design of grid multiscroll chaotic attractors via transformations," International Journal of Bifurcation and Chaos, vol. 25, no. 10, Article ID 1530027, 2015.

[37] V.-T. Pham, C. K. Volos, and S. Vaidyanathan, "Multi-scroll chaotic oscillator based on a first-order delay differential equation," Studies in Computational Intelligence, vol. 581, pp. 59-72, 2015.

[38] J. Lü and G. Chen, "Generating multiscroll chaotic attractors: theories, methods and applications," International Journal of Bifurcation and Chaos, vol. 16, no. 4, pp. 775-858, 2006.

[39] J. M. Muñoz-Pacheco, E. Zambrano-Serrano, O. Félix-Beltrán, L. C. Gómez-Pavón, and A. Luis-Ramos, "Synchronization of PWL function-based 2D and 3D multi-scroll chaotic systems," Nonlinear Dynamics, vol. 70, no. 2, pp. 1633-1643, 2012. 


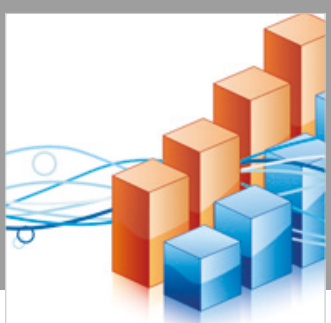

Advances in

Operations Research

\section{-n-m}
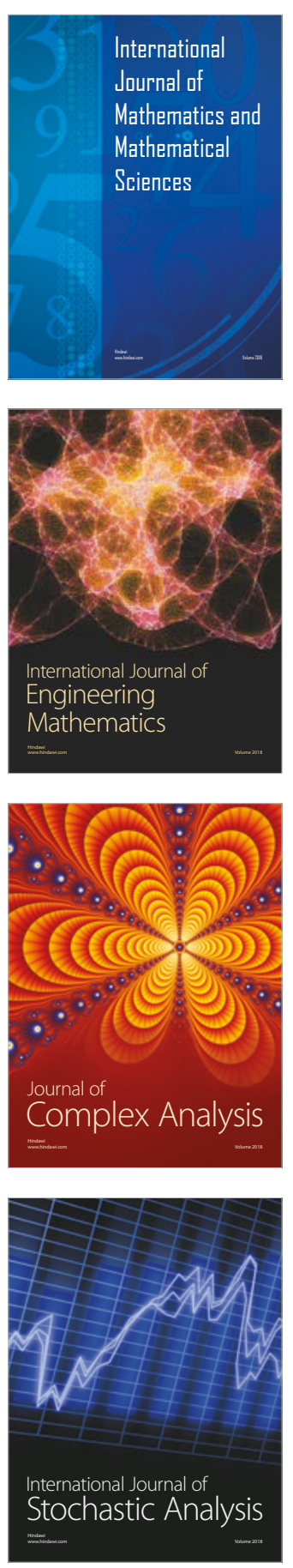
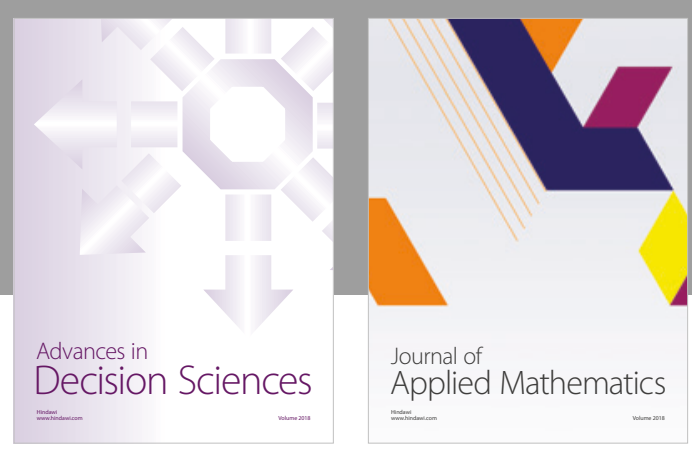

Journal of

Applied Mathematics
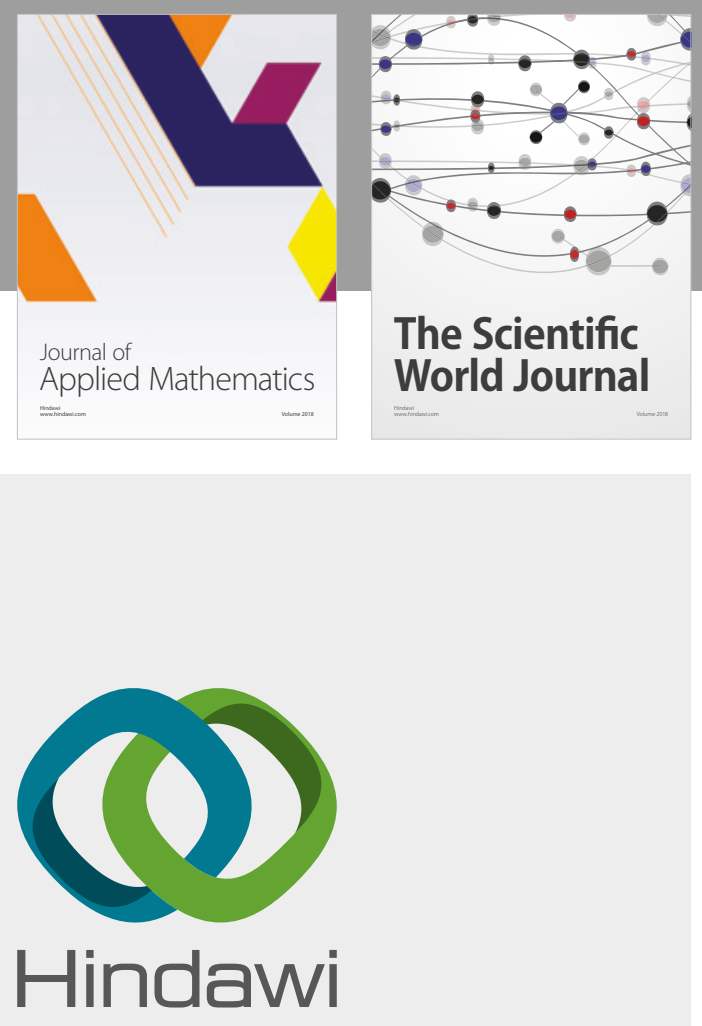

Submit your manuscripts at

www.hindawi.com

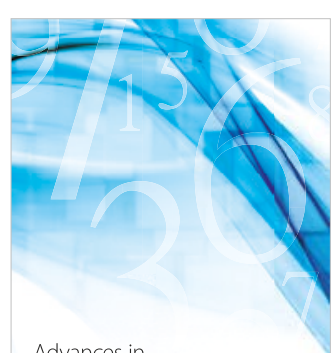

Advances in
Numerical Analysis
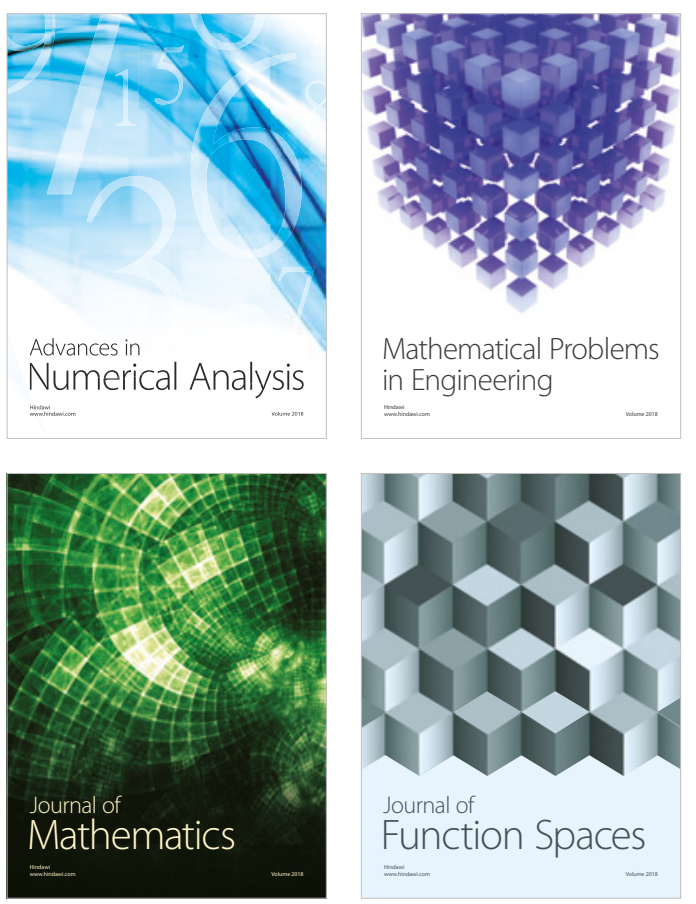

Mathematical Problems in Engineering

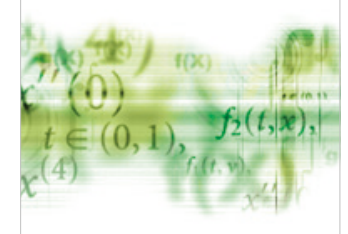

International Journal of

Differential Equations

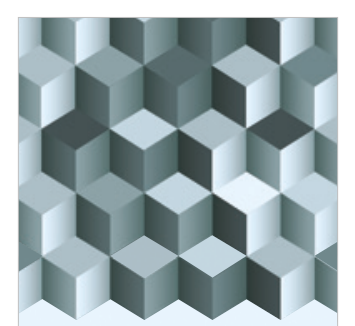

Journal of

Function Spaces
The Scientific

World Journal

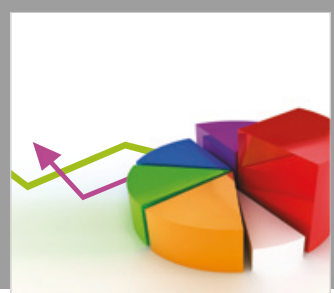

Journal of

Probability and Statistics
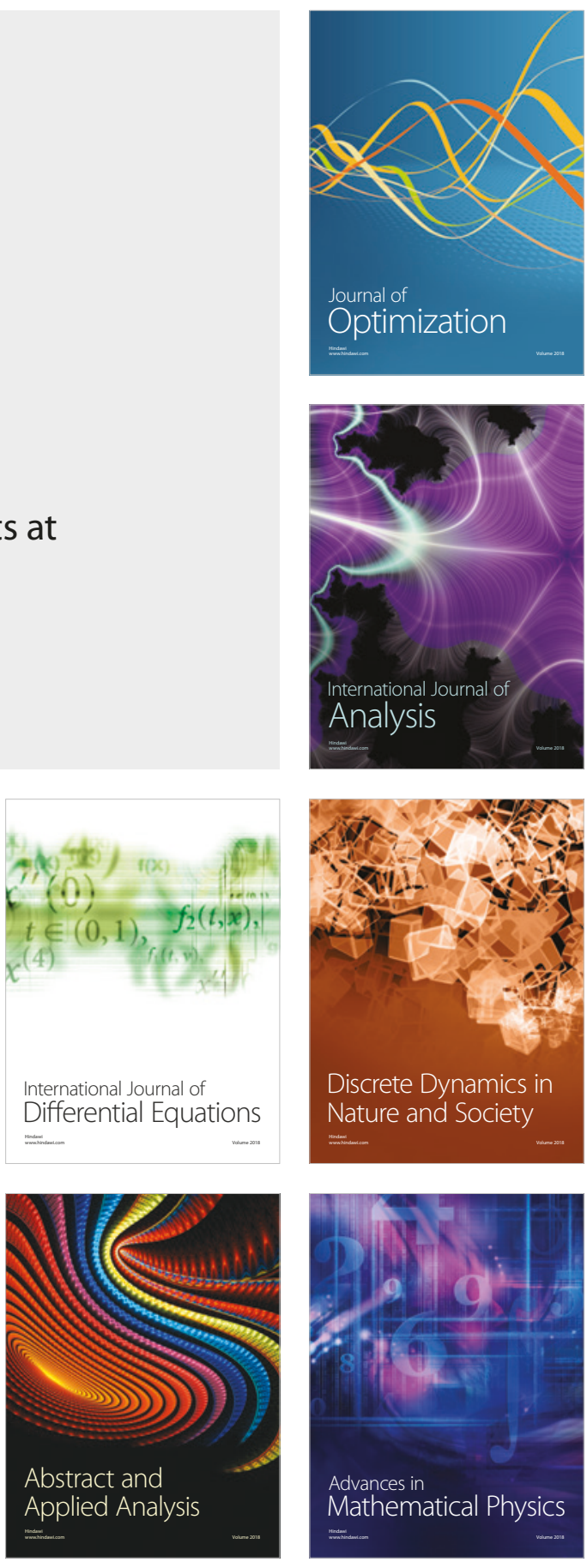